\title{
A Facile One-Pot Synthesis of Alkyl Aryl Sulfides from Aryl Bromides
}

\author{
Jungyeob Ham, Inho Yang, and Heonjoong Kang* \\ Marine Biotechnology Laboratory and Research Institute of Oceanography, School of Earth \\ and Environmental Sciences, Seoul National University, \\ NS-80, Seoul 151-747, Korea.
}

\section{Table of Contents for Supporting Information}

General Considerations: ${ }^{1} \mathrm{H}$ and ${ }^{13} \mathrm{C}$ NMR spectra were recorded on $300 \mathrm{MHz}$ and $500 \mathrm{MHz}$ instruments in $\mathrm{CDCl}_{3}$ or DMSO-d $\mathrm{d}_{6}$, using TMS as the internal standard. Mass spectra were recorded by the electron impact method $(70 \mathrm{eV})$ or chemical ionization method.

Commercially obtained anhydrous THF and reagents were used without further purification.

Copy of ${ }^{1} \mathrm{H},{ }^{13} \mathrm{C}$, and EI mass Spectra of entry $\mathbf{1}$ Copy of ${ }^{1} \mathrm{H},{ }^{13} \mathrm{C}$, and EI mass Spectra of entry 2 Copy of ${ }^{1} \mathrm{H},{ }^{13} \mathrm{C}$, and EI mass Spectra of entry 3 Copy of ${ }^{1} \mathrm{H},{ }^{13} \mathrm{C}$, and EI mass Spectra of entry 4 Copy of ${ }^{1} \mathrm{H},{ }^{13} \mathrm{C}$, and EI mass Spectra of entry 5 Copy of ${ }^{1} \mathrm{H},{ }^{13} \mathrm{C}$, and EI mass Spectra of entry 6 Copy of ${ }^{1} \mathrm{H},{ }^{13} \mathrm{C}$, and EI mass Spectra of entry 7 Copy of ${ }^{1} \mathrm{H},{ }^{13} \mathrm{C}$, and EI mass Spectra of entry 8 Copy of ${ }^{1} \mathrm{H},{ }^{13} \mathrm{C}$, and EI mass Spectra of entry 9 Copy of ${ }^{1} \mathrm{H},{ }^{13} \mathrm{C}$, and EI mass Spectra of entry $\mathbf{1 0}$ Copy of ${ }^{1} \mathrm{H},{ }^{13} \mathrm{C}$, and EI mass Spectra of entry $\mathbf{1 1}$ Copy of ${ }^{1} \mathrm{H},{ }^{13} \mathrm{C}$, and EI mass Spectra of entry 12 Copy of ${ }^{1} \mathrm{H},{ }^{13} \mathrm{C}$, and EI mass Spectra of entry $\mathbf{1 3}$ Copy of ${ }^{1} \mathrm{H},{ }^{13} \mathrm{C}$, and EI mass Spectra of entry 14 Copy of ${ }^{1} \mathrm{H},{ }^{13} \mathrm{C}$, and EI mass Spectra of entry $\mathbf{1 5}$ Copy of ${ }^{1} \mathrm{H},{ }^{13} \mathrm{C}$, and EI mass Spectra of entry $\mathbf{1 6}$ Copy of ${ }^{1} \mathrm{H},{ }^{13} \mathrm{C}$, and EI mass Spectra of entry 17
S-1 $\sim$ S-3
S-4 $\sim$ S-6
$\mathrm{S}-7 \sim \mathrm{S}-9$
S-10 S-12
$\mathrm{S}-13 \sim \mathrm{S}-15$
S-16 S-18
$\mathrm{S}-19 \sim \mathrm{S}-21$
$\mathrm{S}-22 \sim \mathrm{S}-24$
$\mathrm{S}-25 \sim \mathrm{S}-27$
S-28 S-30
$\mathrm{S}-31 \sim \mathrm{S}-33$
$\mathrm{S}-34 \sim \mathrm{S}-36$
S-37 S-39
$\mathrm{S}-40 \sim \mathrm{S}-42$
$\mathrm{S}-43 \sim \mathrm{S}-45$
$\mathrm{S}-46 \sim \mathrm{S}-48$
S-49 S-51 


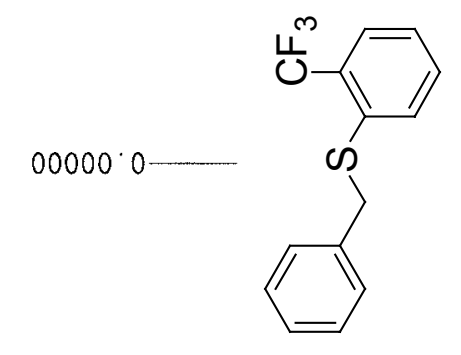

GESES

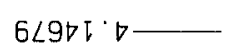

टGEE己 $\angle$

GO8E $\angle \neg$

OSGจ2 $\angle \neg$

IIES己 $\angle \neg)$

$\left.\varepsilon \angle \angle I C C^{\circ} \angle \neg\right)$

$\angle L$ LO' $\angle \neg V$

โวG82

จIE6己

EIटOE'

$098 \angle E^{\circ} \angle$

Іटा6E $\angle$

8टEट9 $\angle$
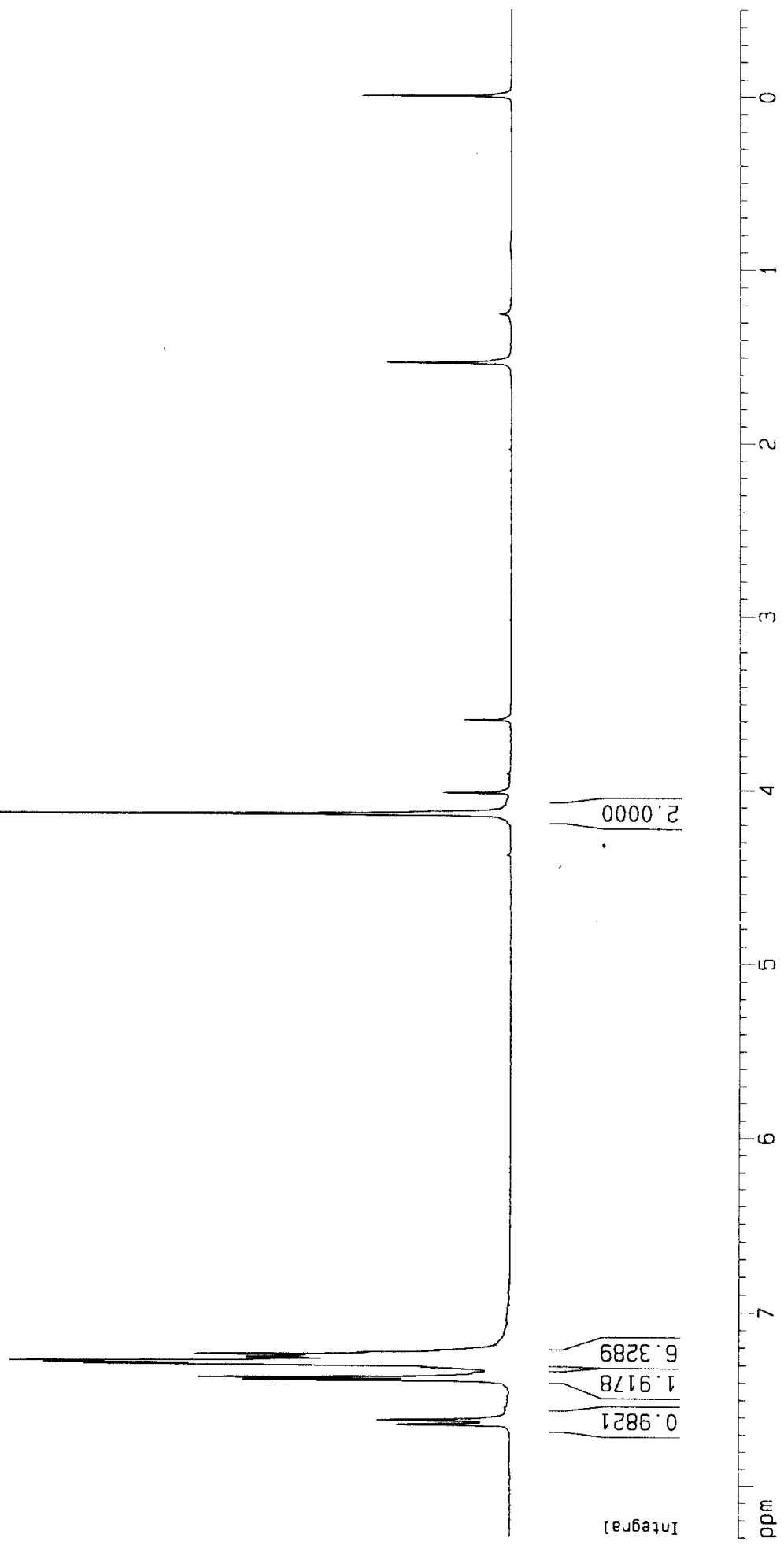

S-1 


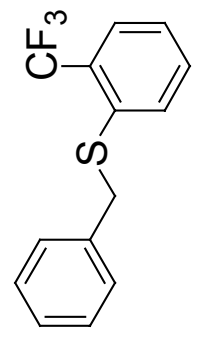

$98 \angle \cdot 6 E$
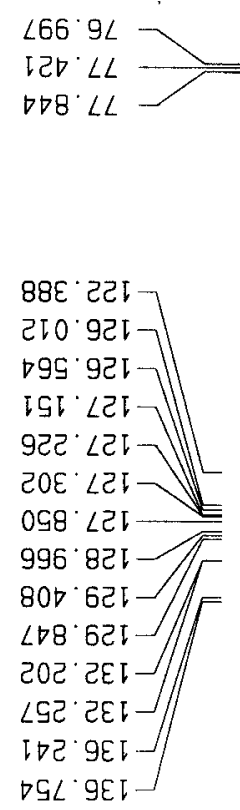

wdd 


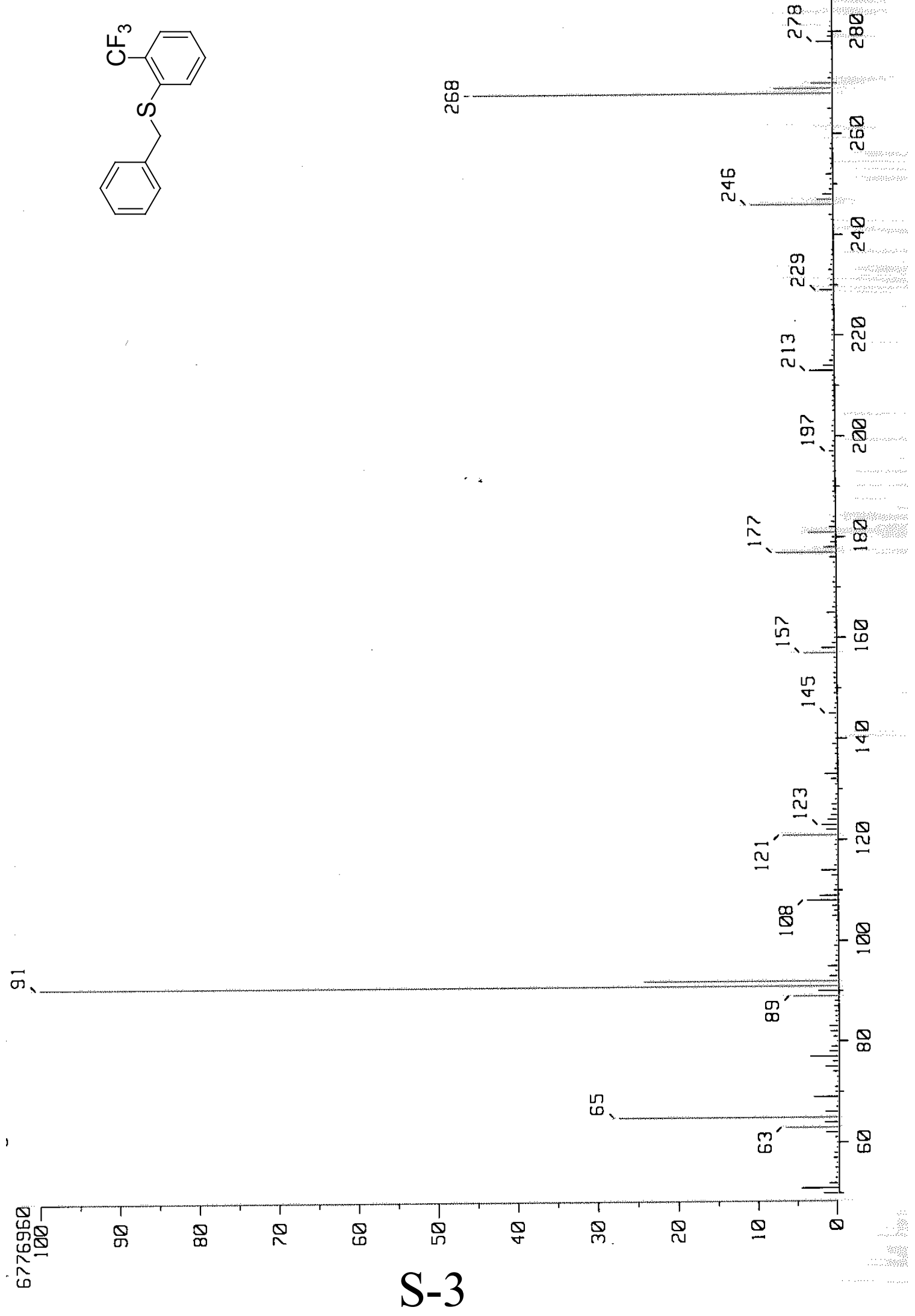




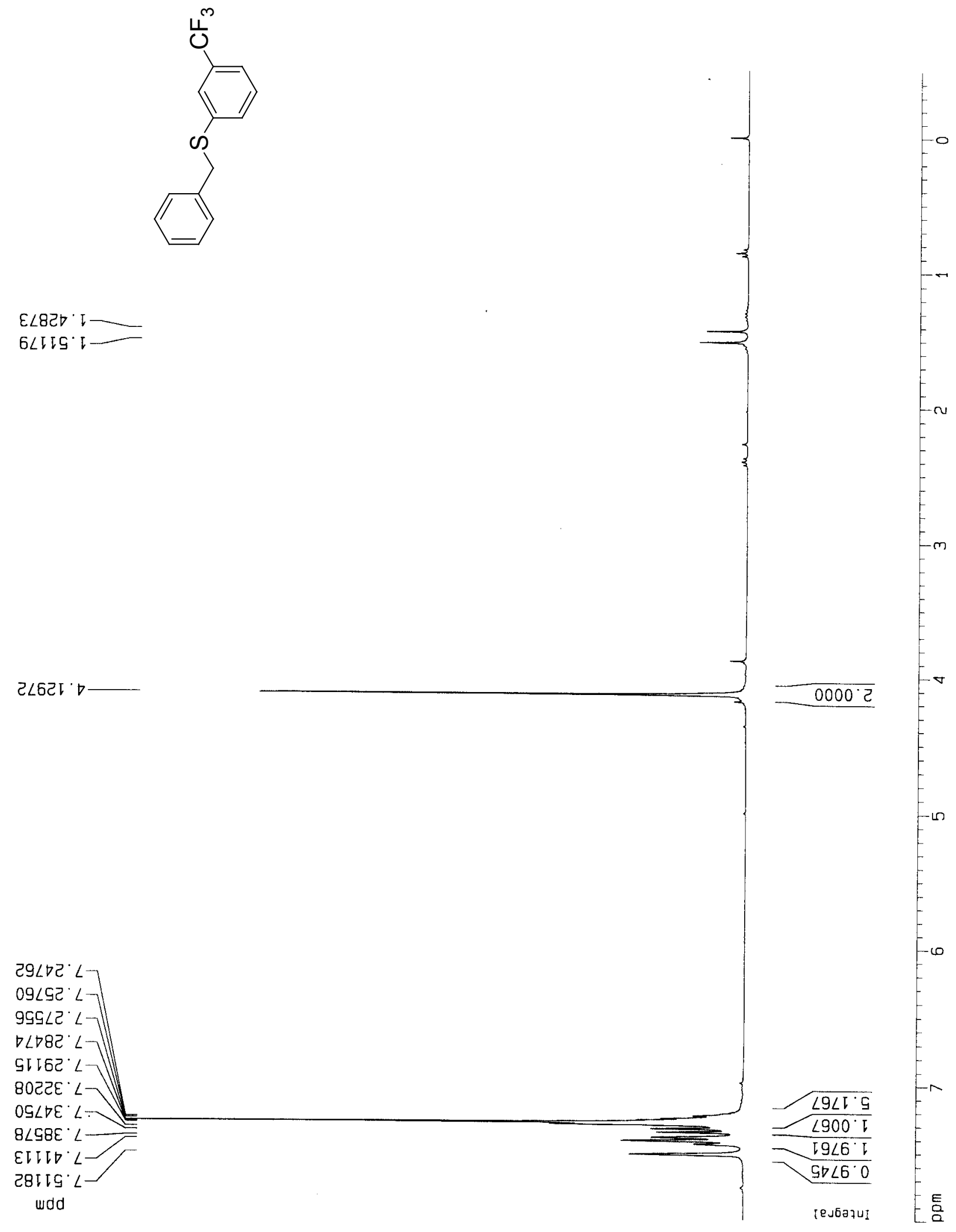




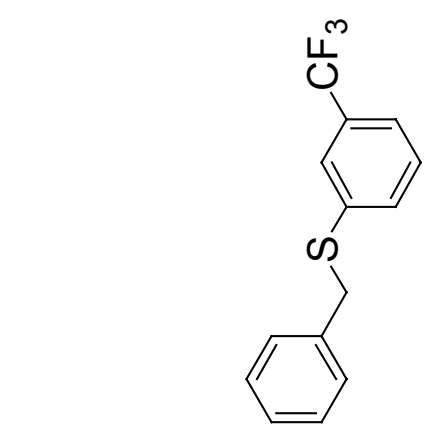

$\angle 60^{\circ} 6 E$
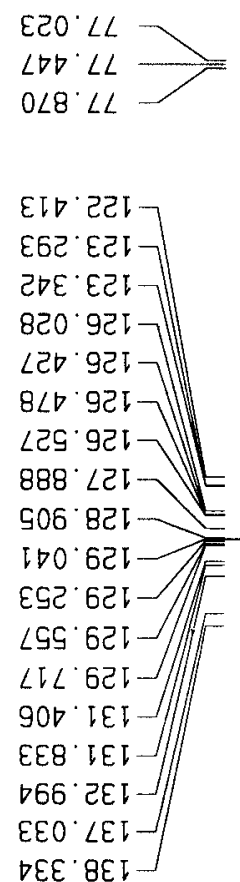

$\triangle E E^{\prime} \mathrm{BE} \mathrm{L}$

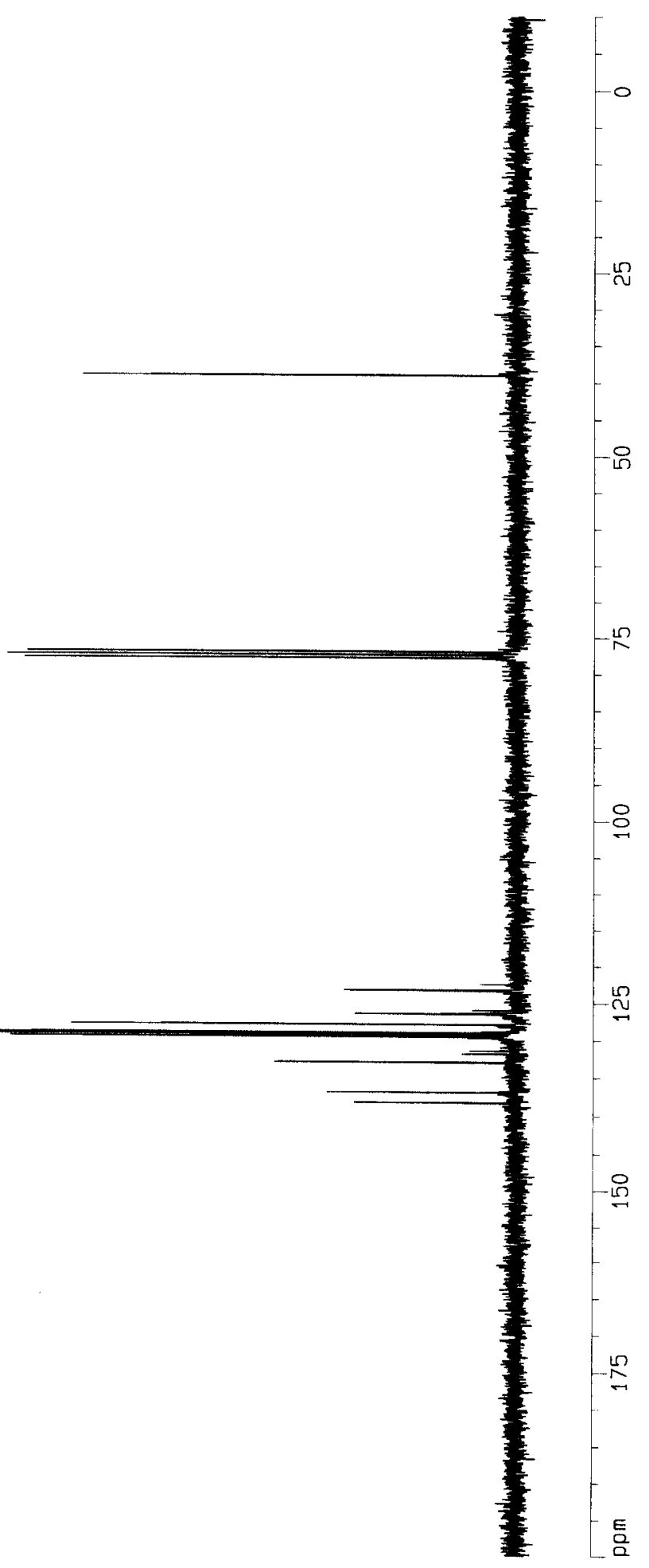

\section{S-5}




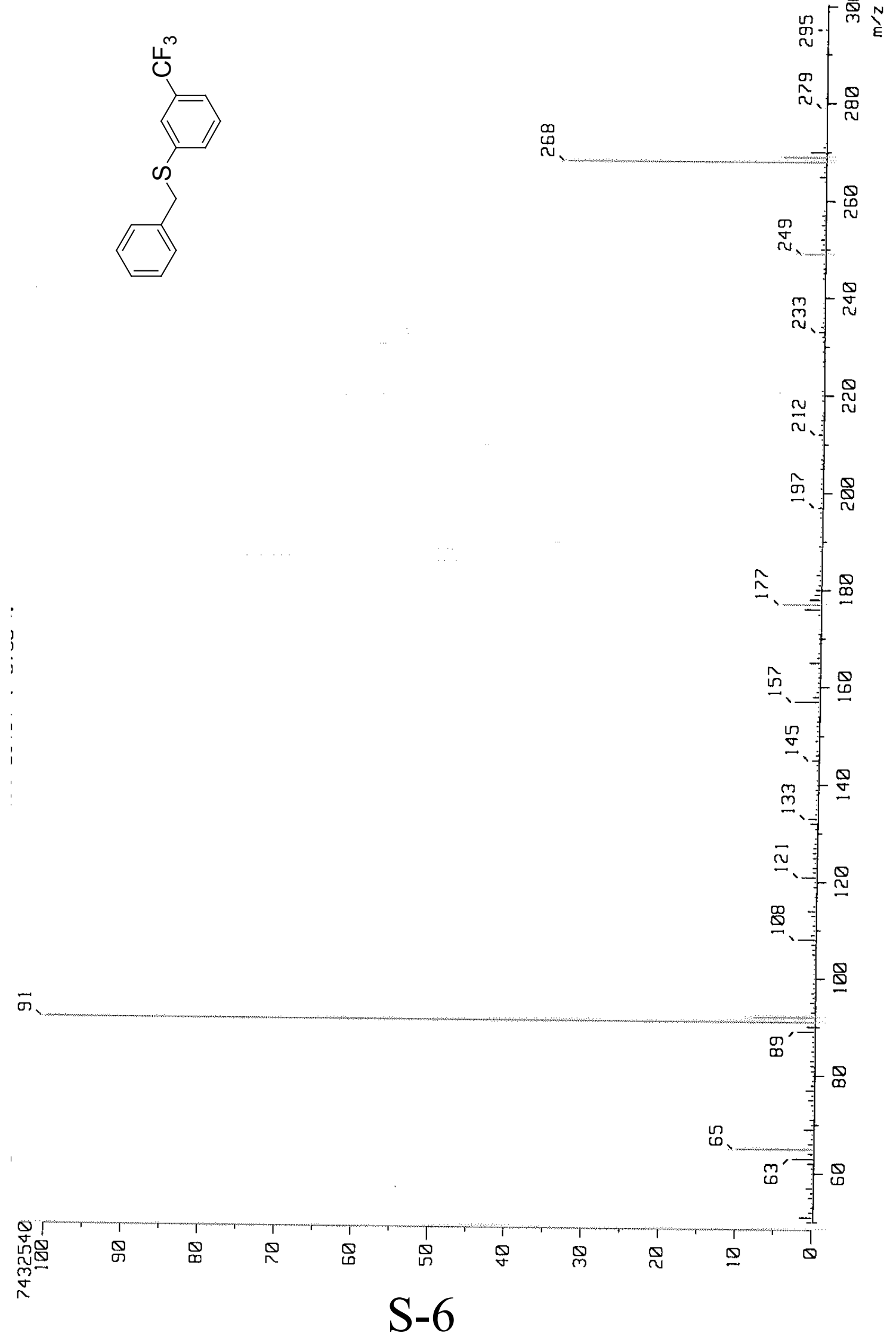




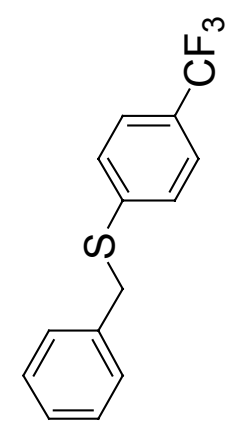

ISSDS

โटE6レ・
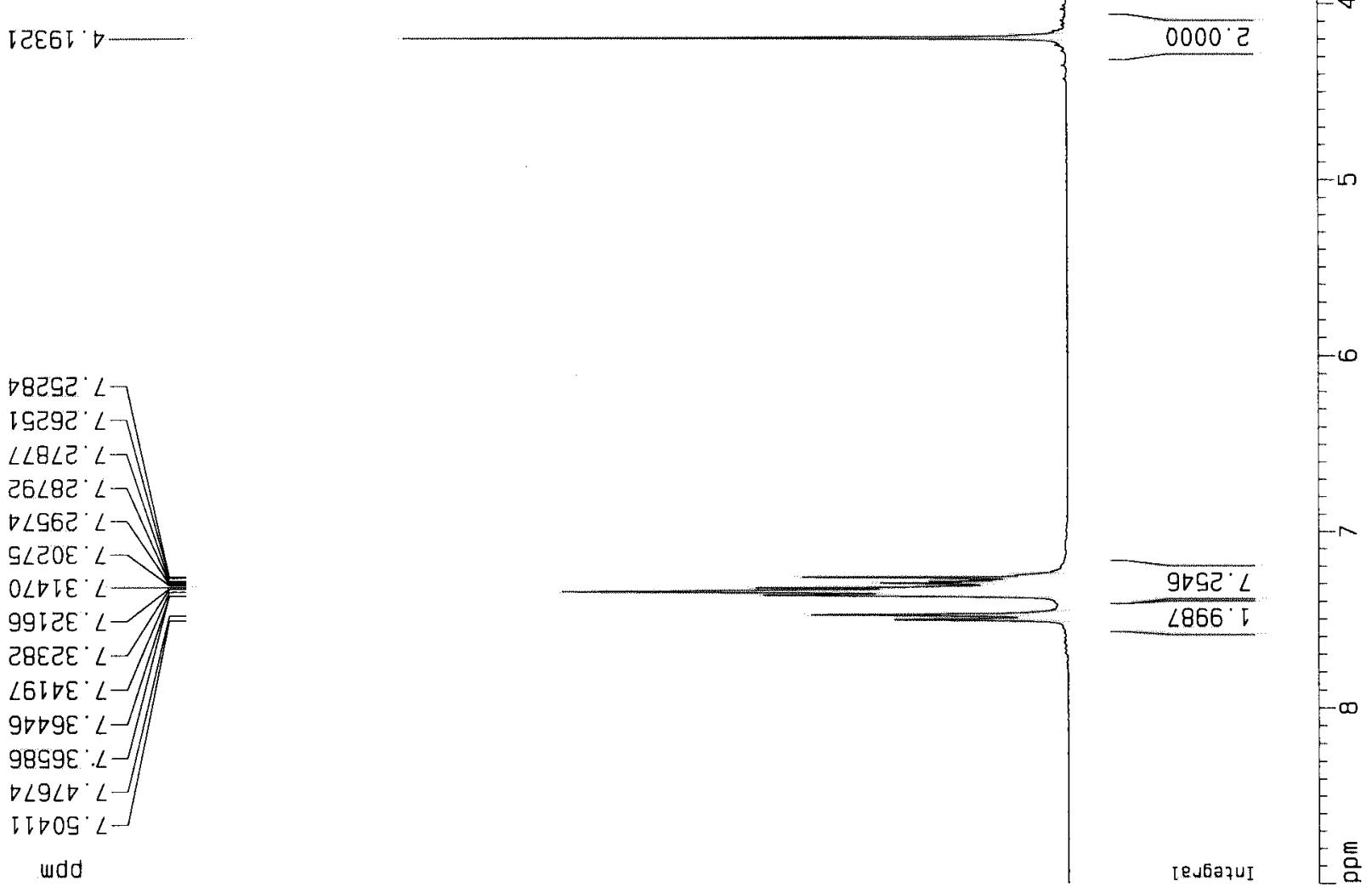


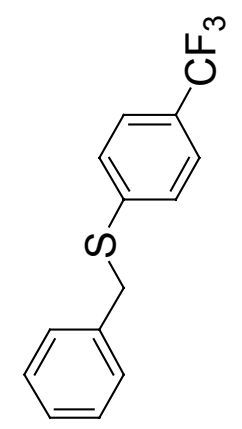

$880^{\circ} 8 \varepsilon$

$866^{\circ} 9 L$

टटD $\angle L$

$9 \nabla 8^{\circ} L L$

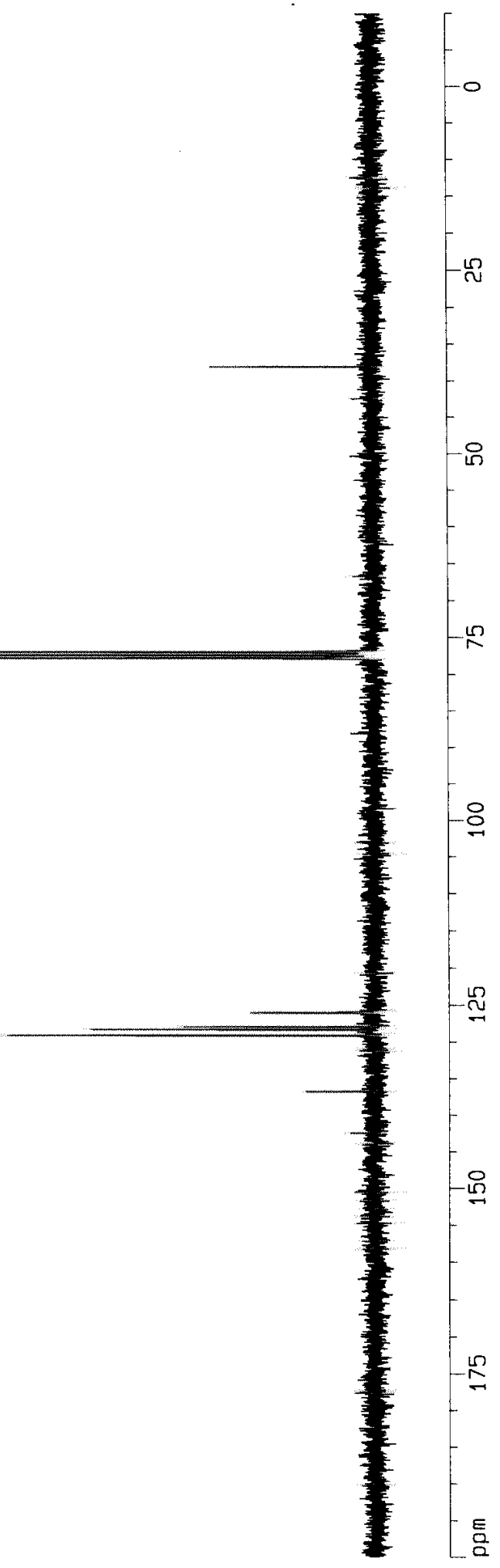

$\angle 86^{\circ}$ 다

8E0 9ट

SE6 $\angle 2 \downarrow$

टाह'8टा

$660^{\circ}$ 6己

6EI 6टा

GSL' $9 E L$ 


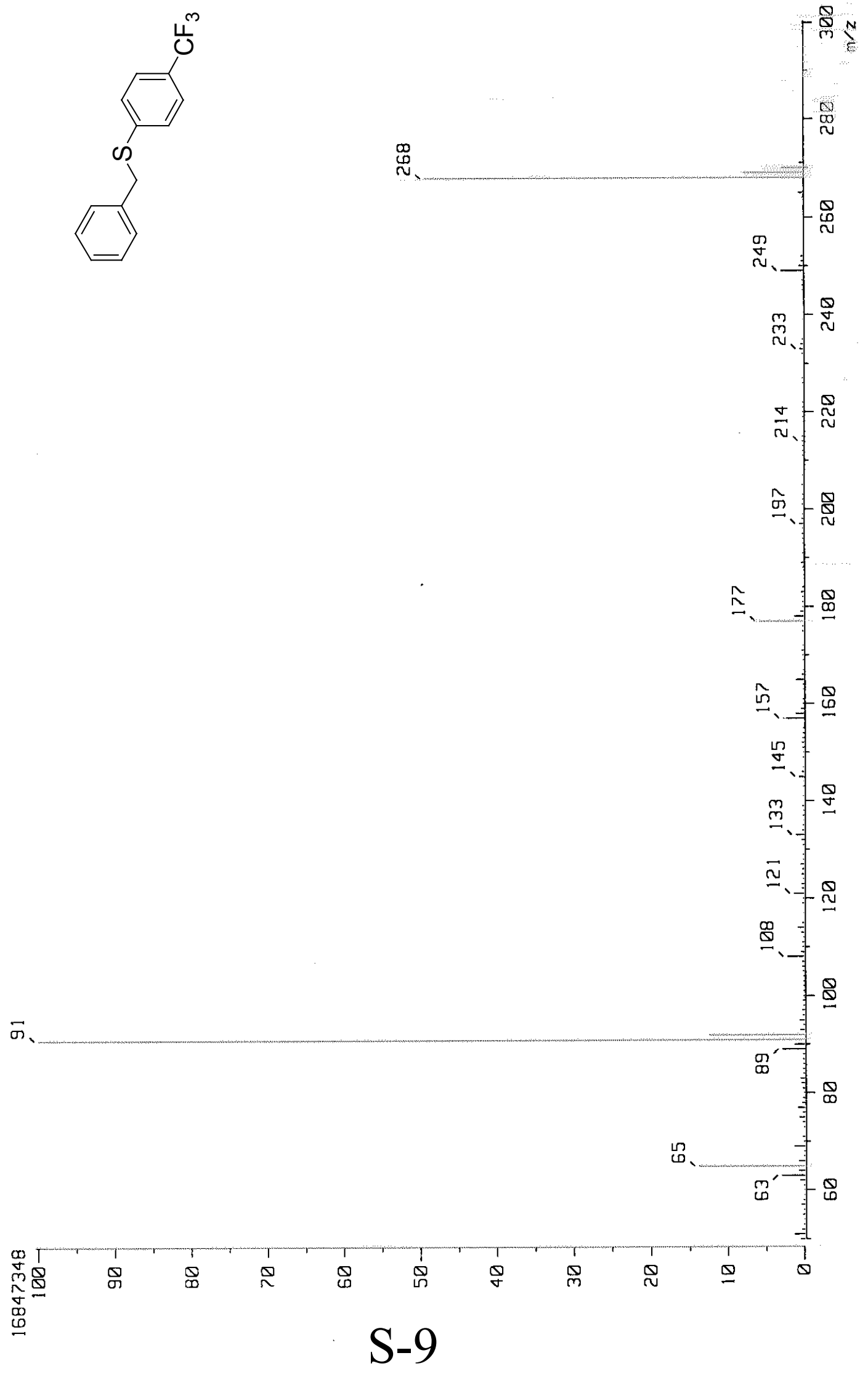




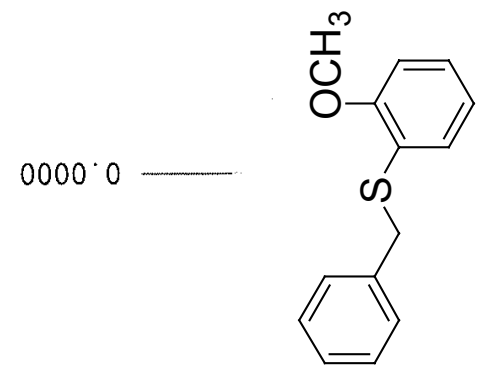

$G \angle D G^{\circ} \downarrow$

$\varepsilon \angle \angle 8^{\circ} \varepsilon$

I880 $\mathrm{O}$
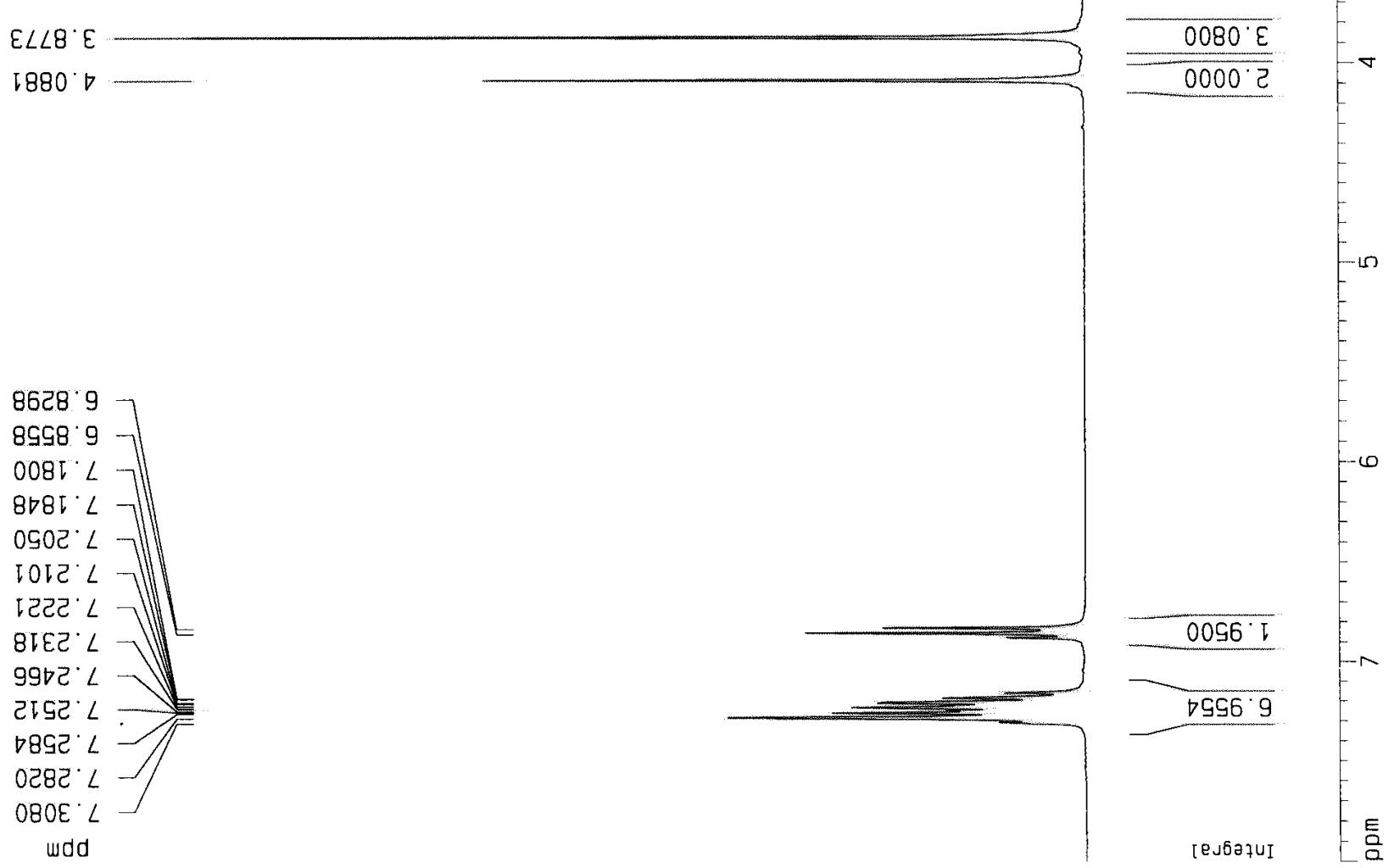


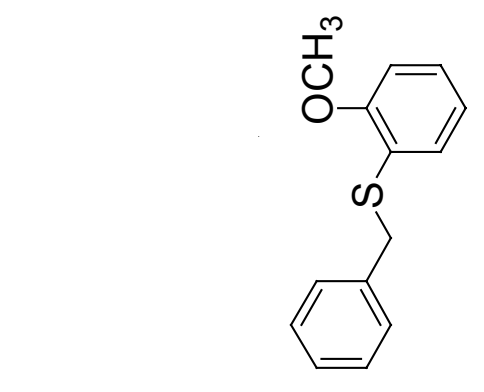

$2 \angle 9^{\circ} \angle E$

981'99

$\angle 10^{\circ} \angle L$
$1 D 0^{\circ} \angle L$
$\square 98^{\circ} \angle L$

688 아 -

$88 \varepsilon^{\circ}$ ᄂ

GE8 $\forall ट \tau$

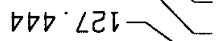

टट0. 8टा

$06 \angle$ : 821

与6ट 6टा

$\checkmark 88^{\circ}$ OE I

OZ6 $\angle E$ I

$S \angle 6^{\circ} \angle G \downarrow$

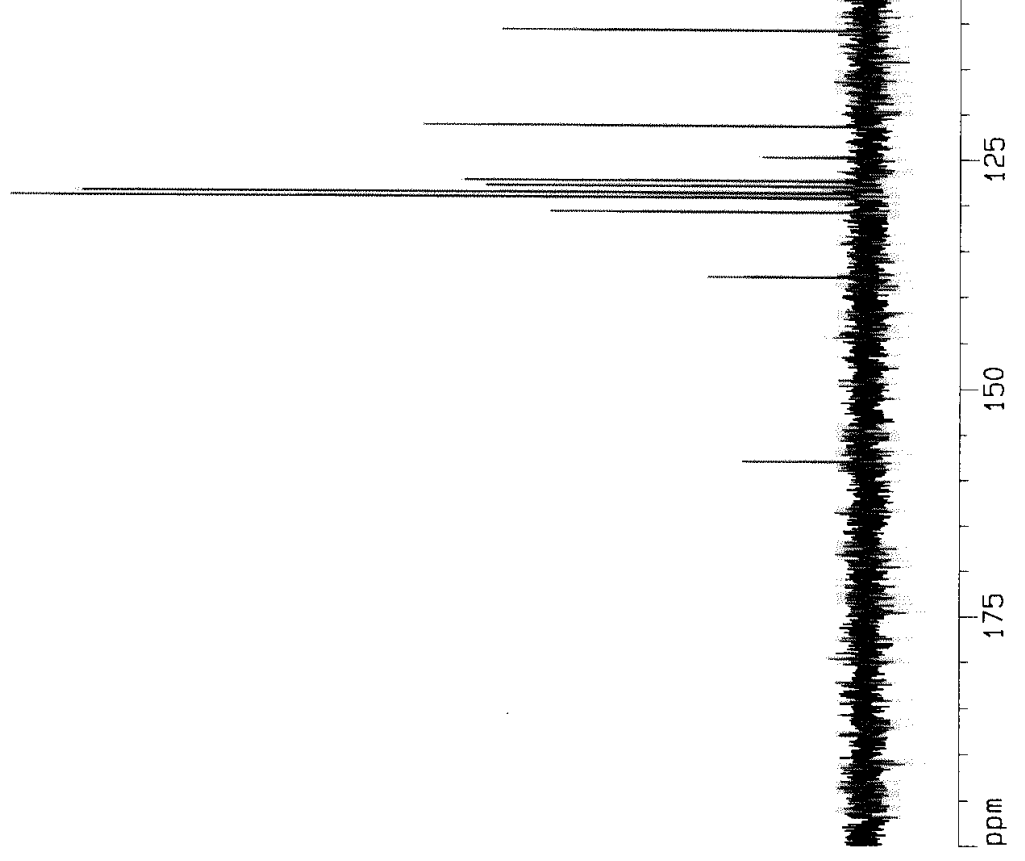

S-11 


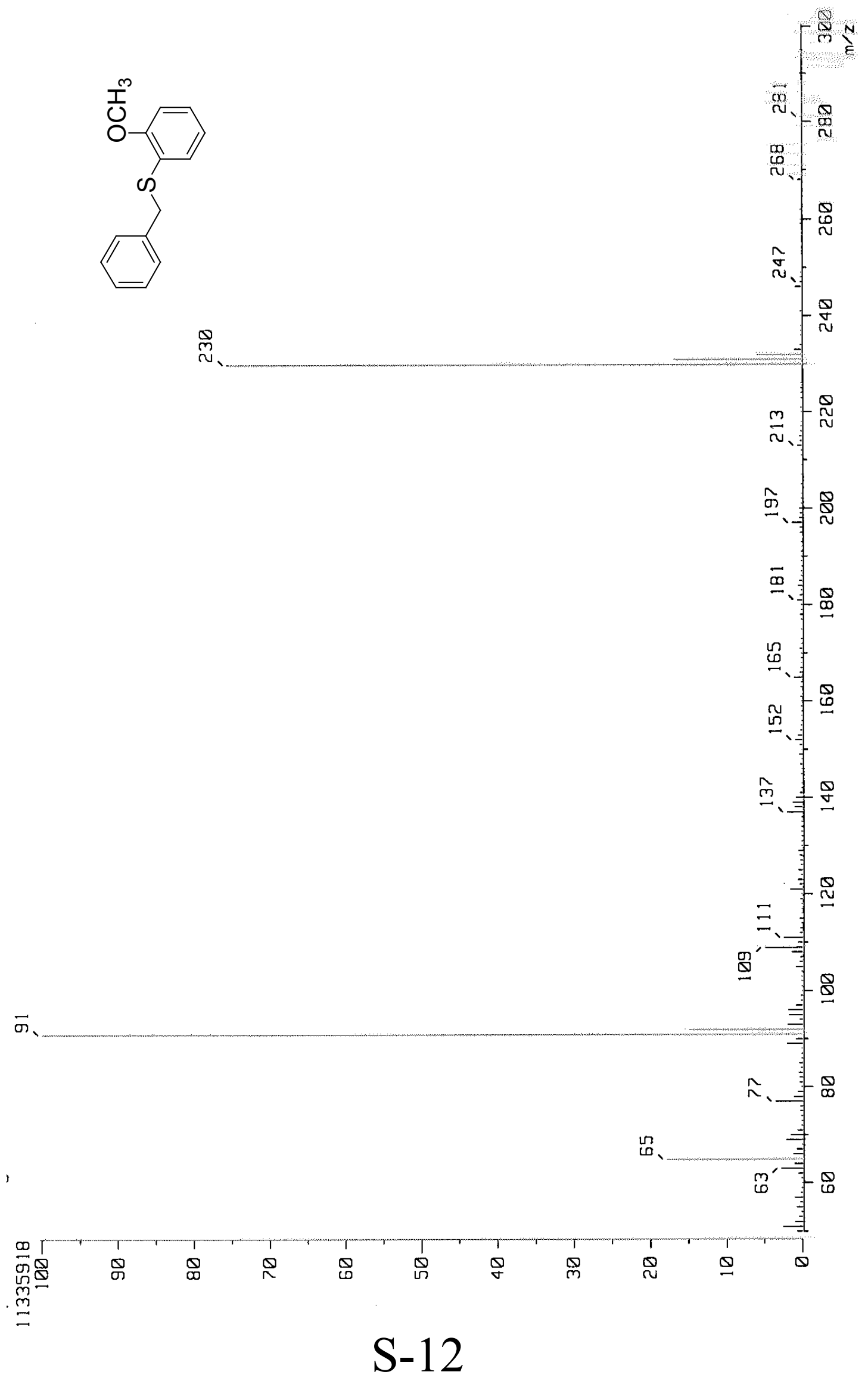




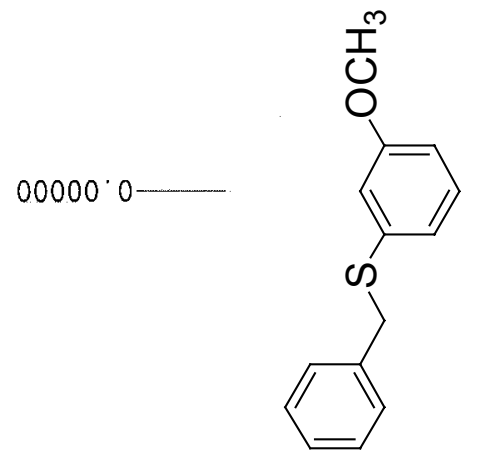

ISLES $\leftarrow$

$065 \angle L^{\circ} \varepsilon$

$902 \varepsilon L^{\circ} \varepsilon$

$\varepsilon I \angle I I^{\circ} \triangleright$
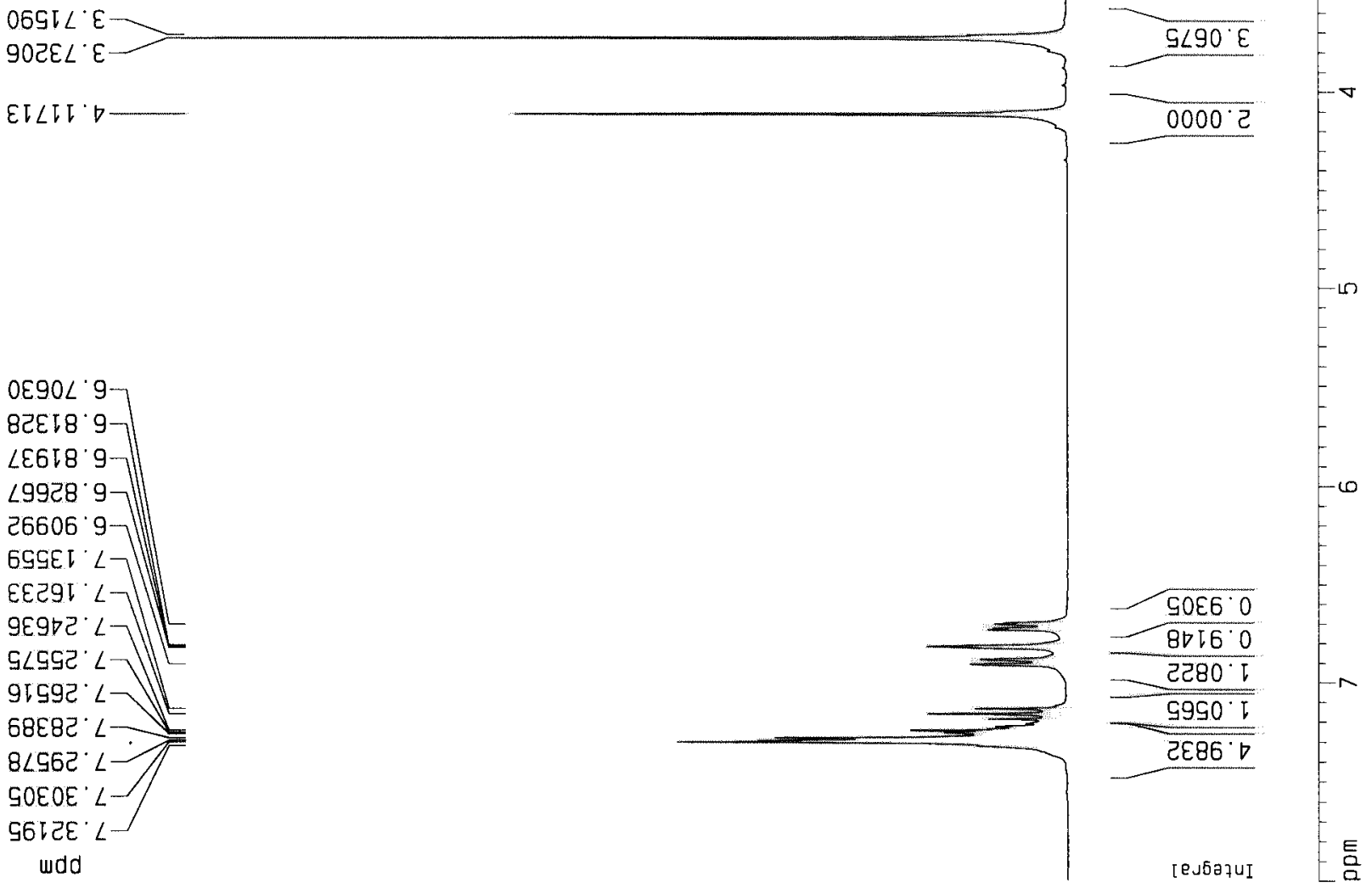


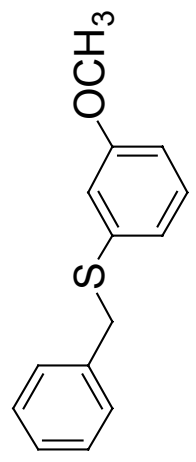

ISE' $6 E$

829.99

$500^{\circ} \angle L$
$620^{\circ} \angle L$
$298^{\circ} \angle L$

SE9 己I -

EOC'GI

E9Гटटा

$669^{\circ} \angle 25$

916.8टा

6Еट" 6टโ

GDO OEI

$608 \cdot \angle E I$

EDI $B E \downarrow$

$\angle E I \cdot 09 I$

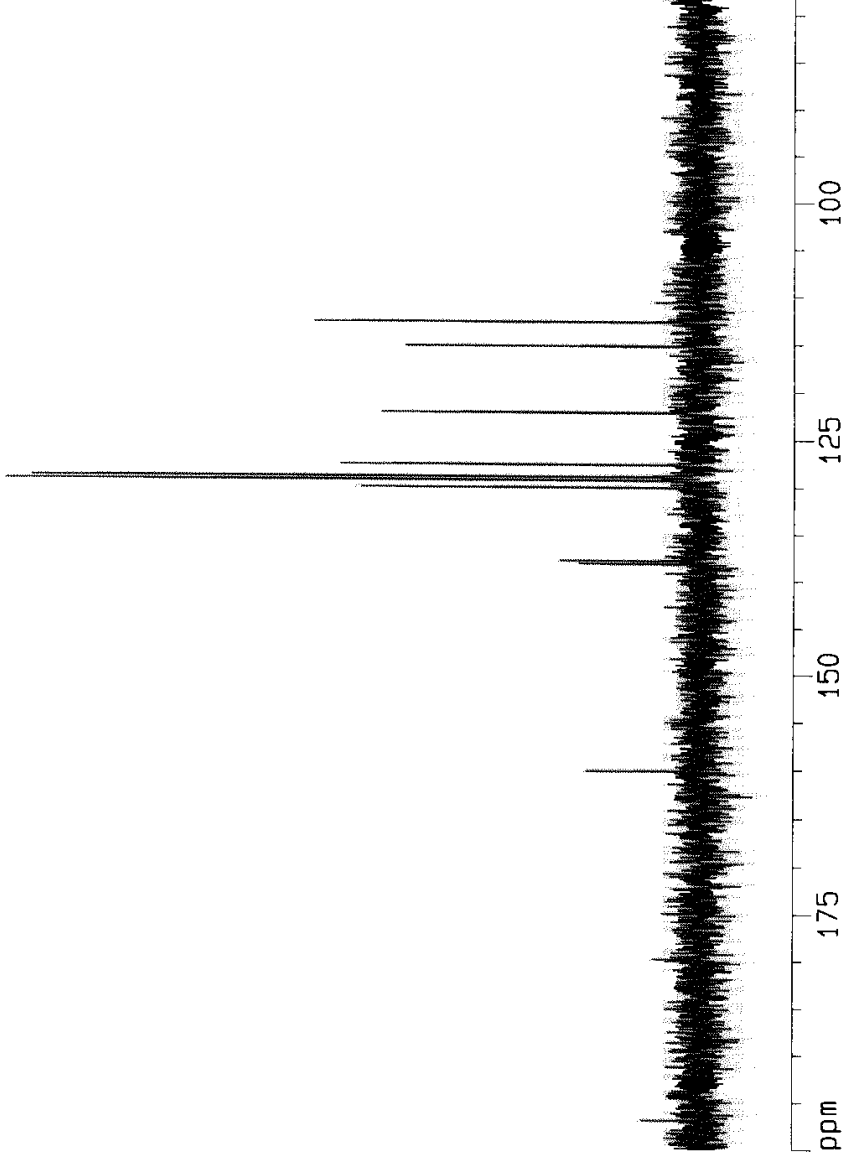

wdd 


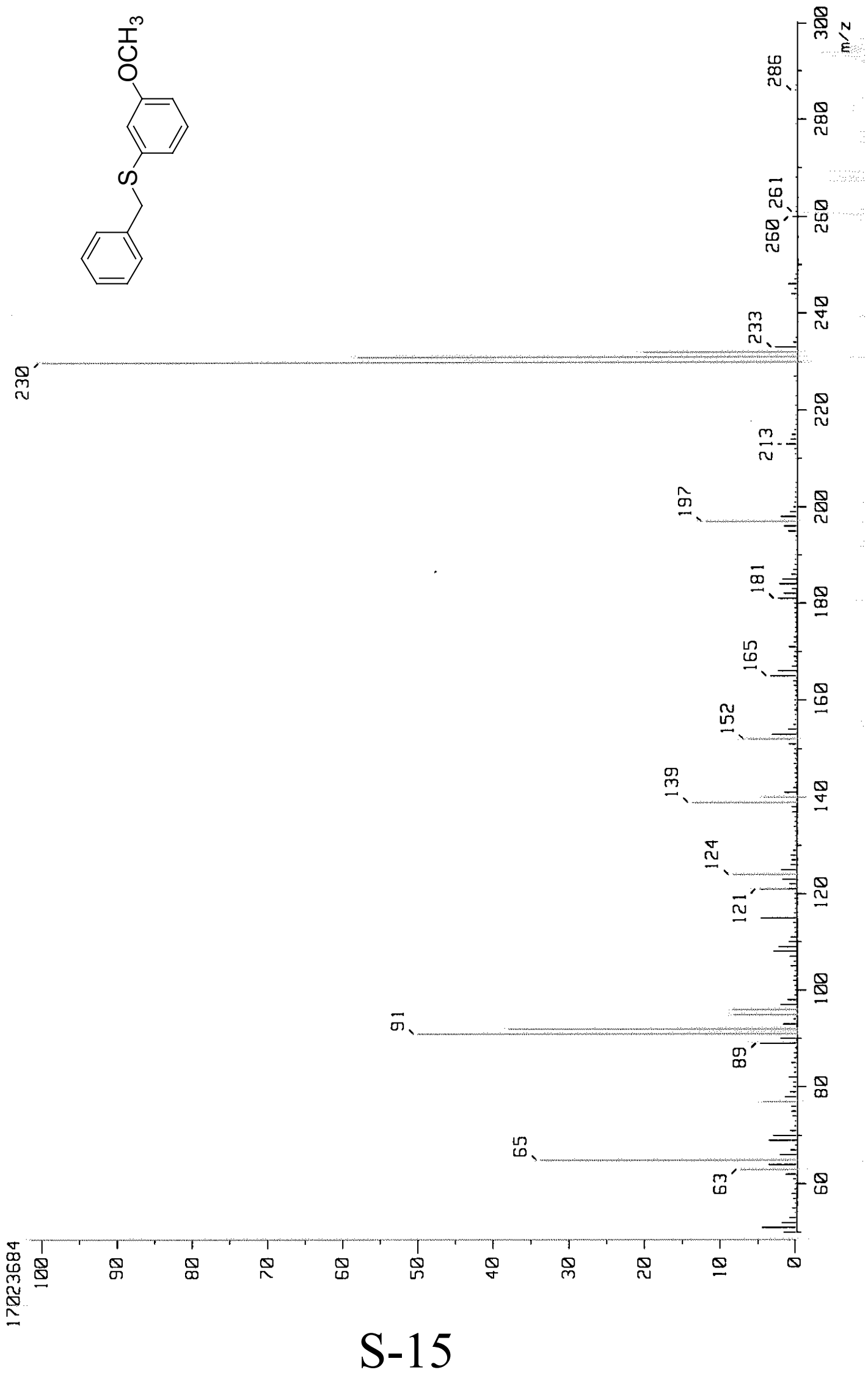




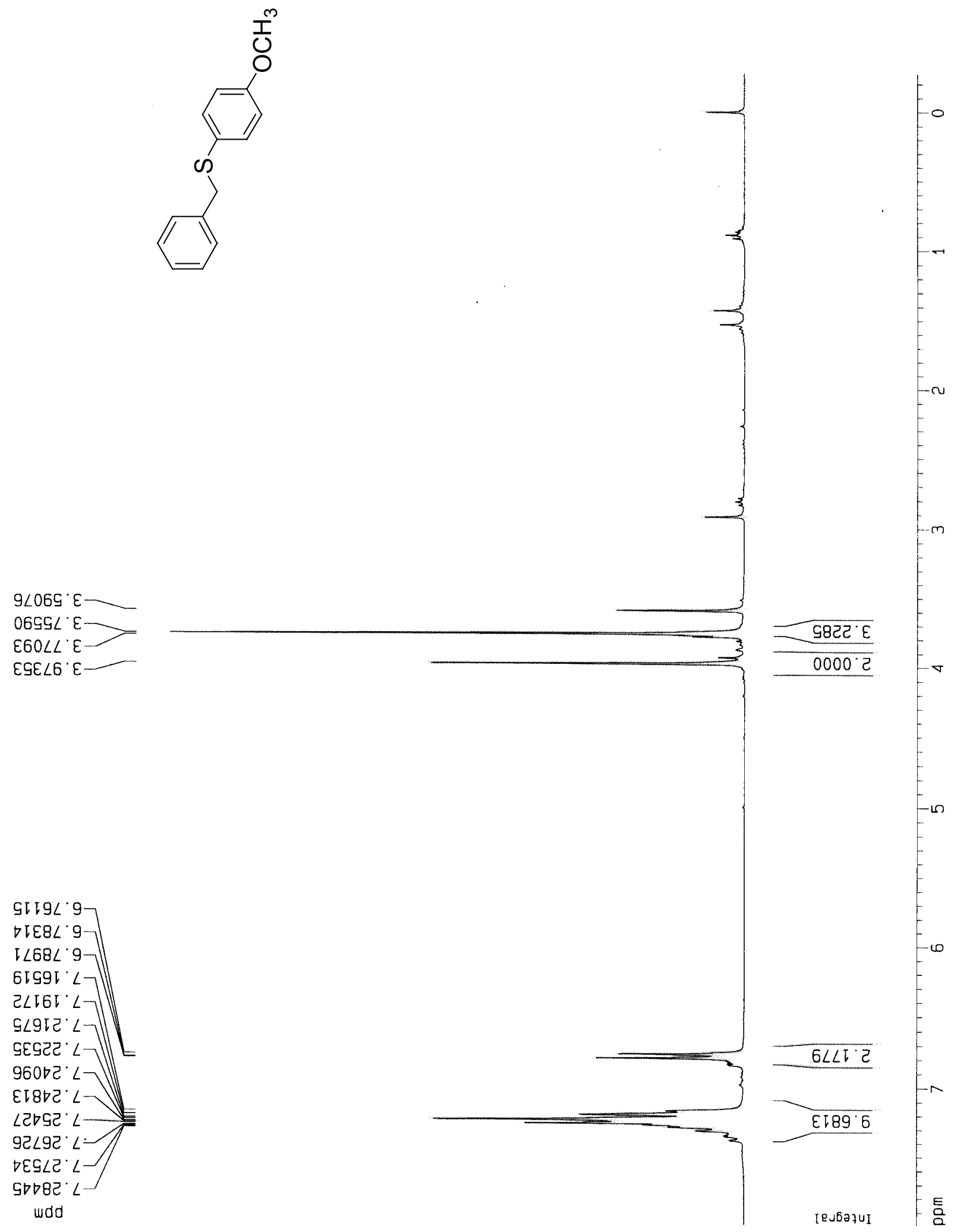




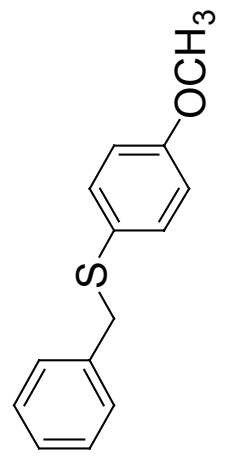

$9 \nabla 9^{\circ} \sqcup \square-$
$8 L \angle E D=$

IL $\mathrm{GS}$

$190^{\circ} \angle L$

$\nabla \angle \nabla^{\circ} \angle L$

$868^{\circ} \mathrm{LL}$
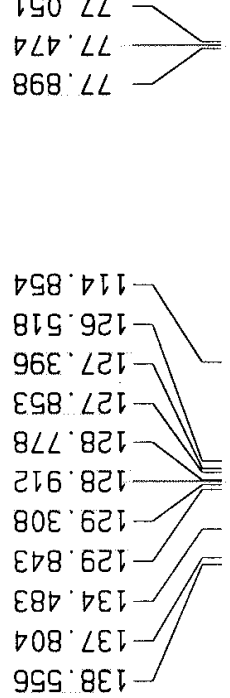

LE9 6GI

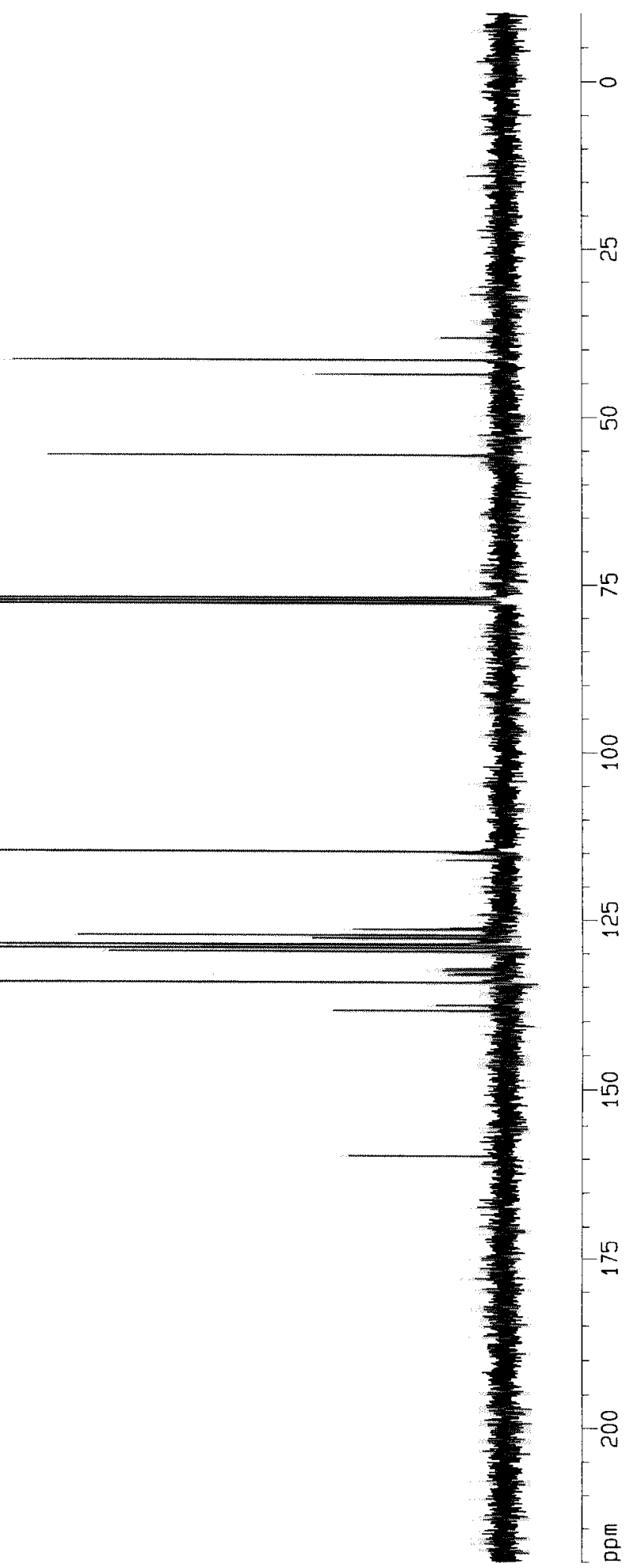




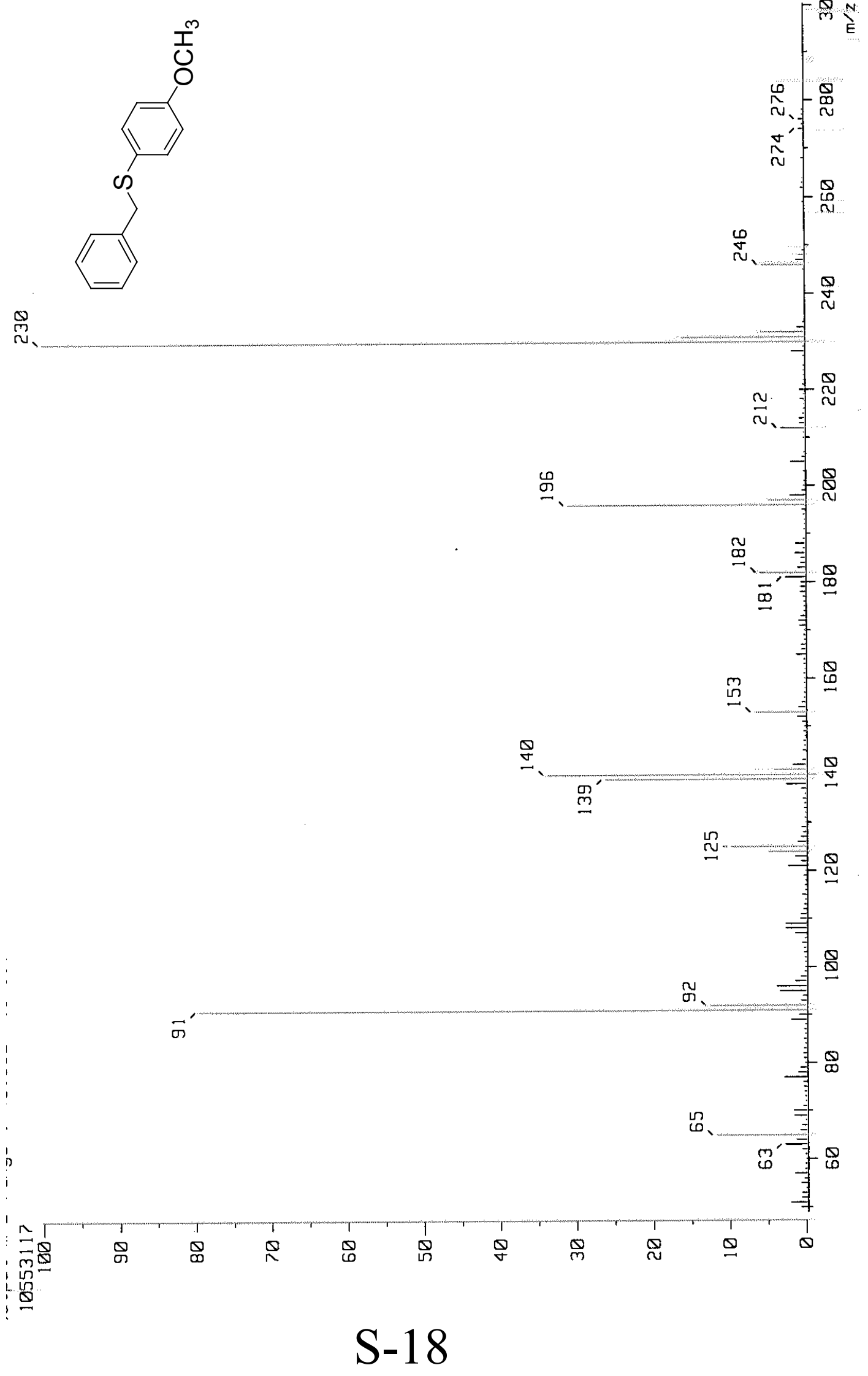




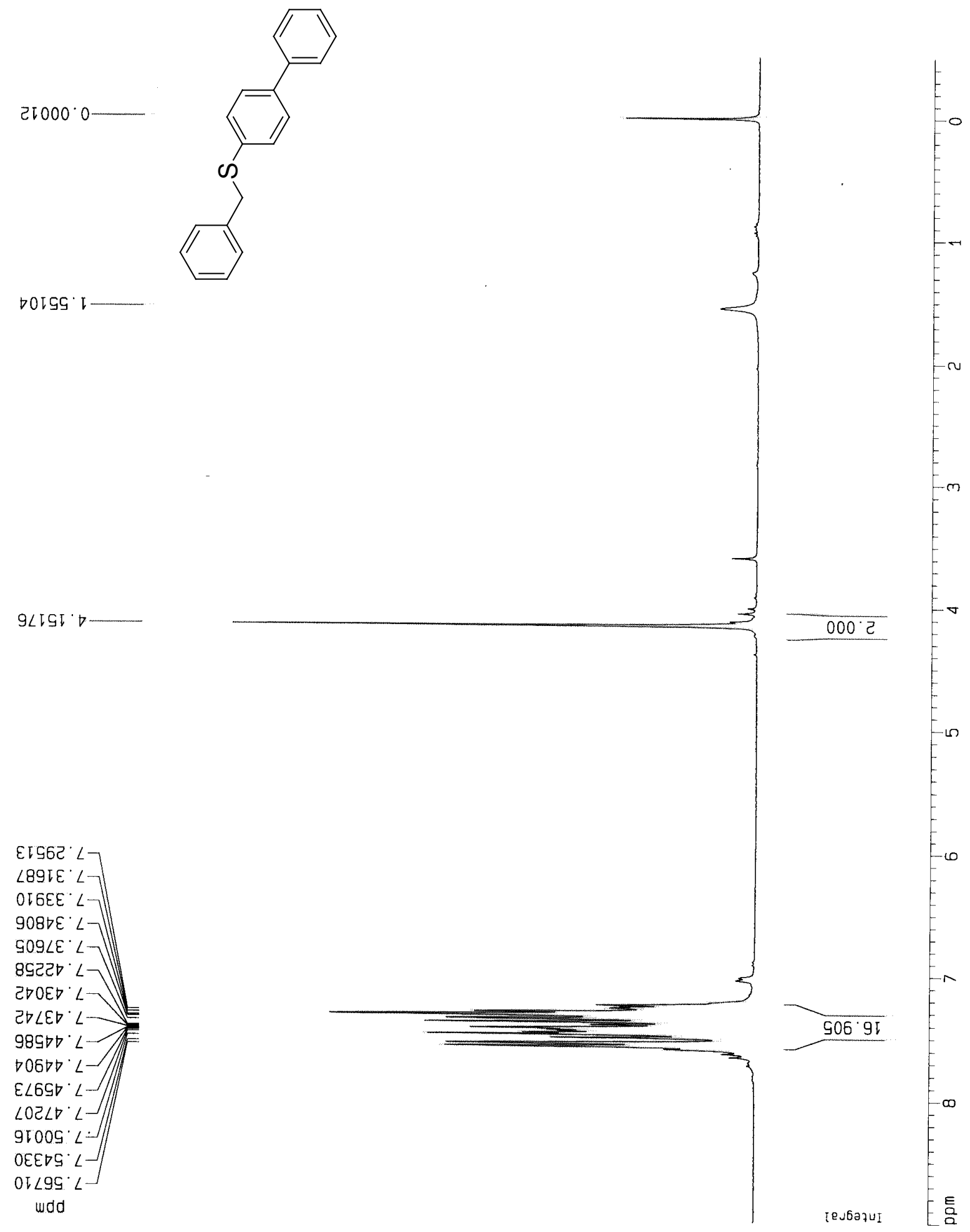




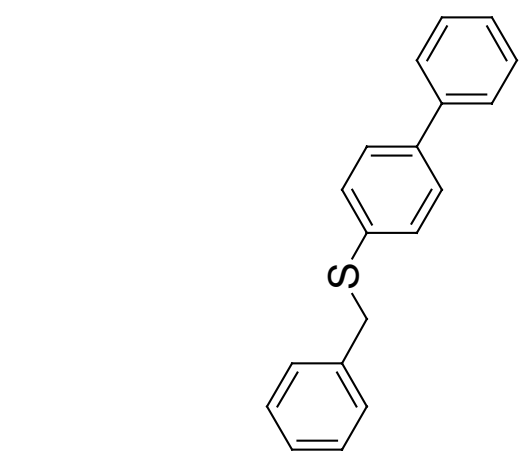

6פจ $6 E$

EIO $\angle L$
$9 E D^{\circ} \angle L$
$698^{\circ} \angle L$

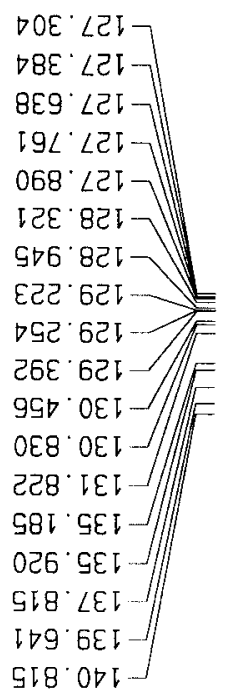

udd 


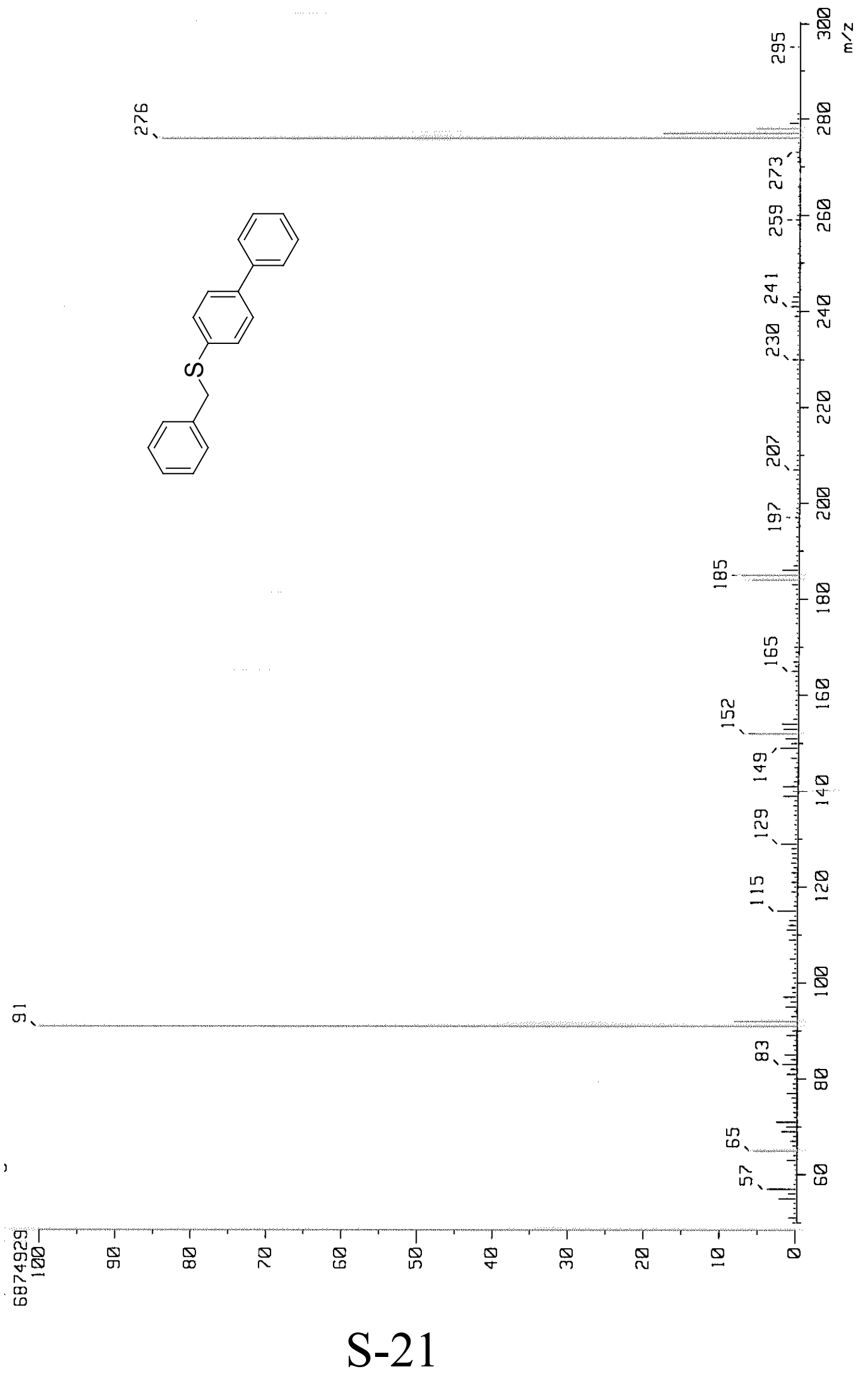



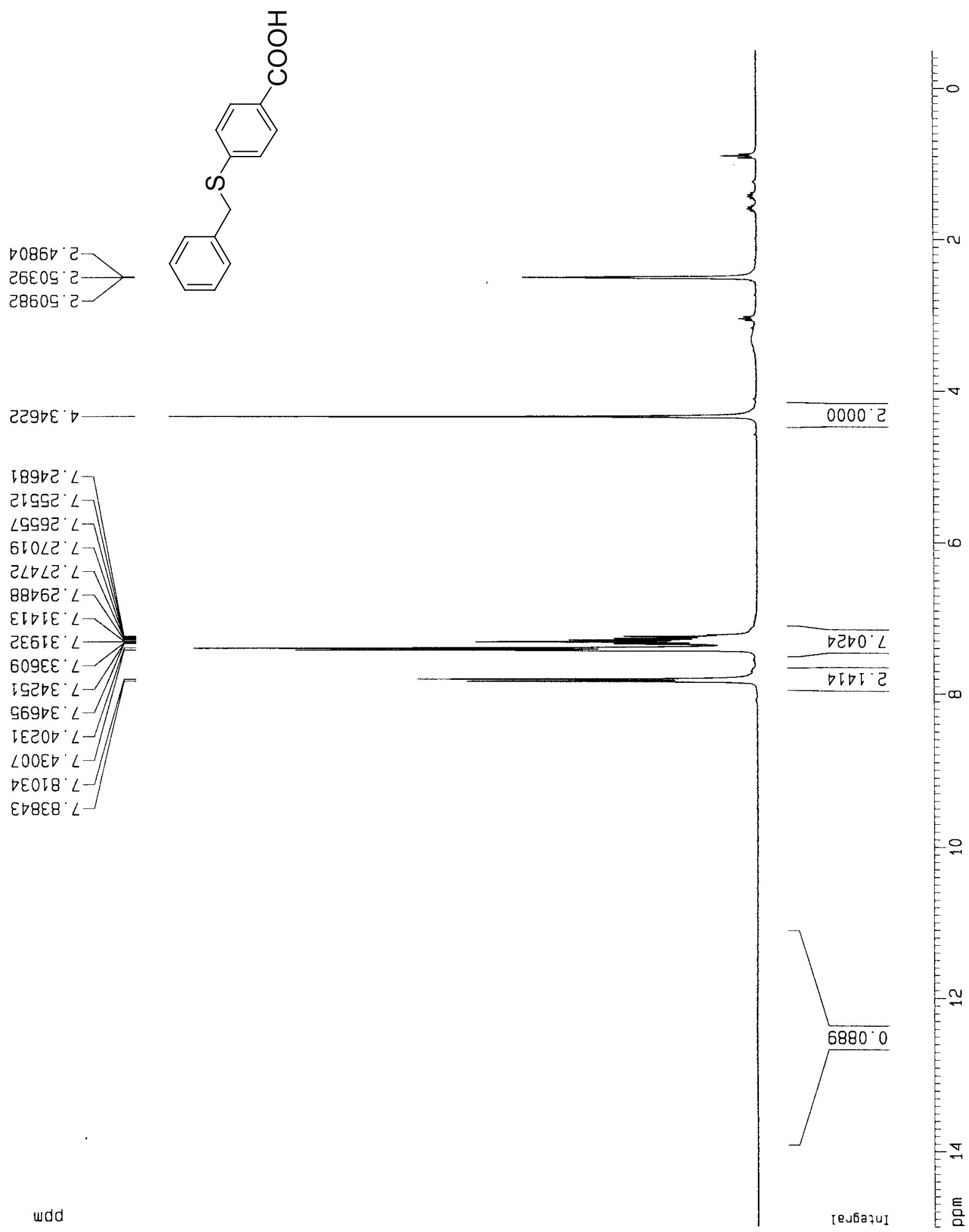


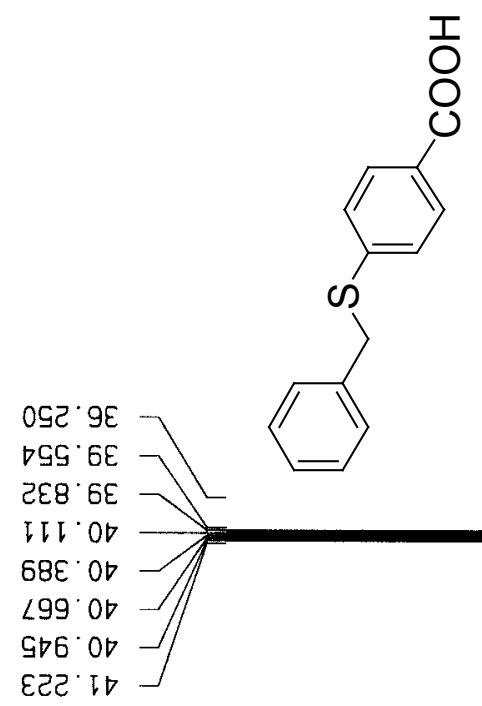

टOE $\angle 2 T$

$880^{\circ} 82 \mathrm{t}$

029.825

$\triangle E E \cdot 62$

$\angle L \angle C L$

6SG OEI

$699^{\circ} \angle E T-$

$969^{\circ} \varepsilon \nabla I-$

$\nabla E 8^{\circ} \angle 9 L$

wdd

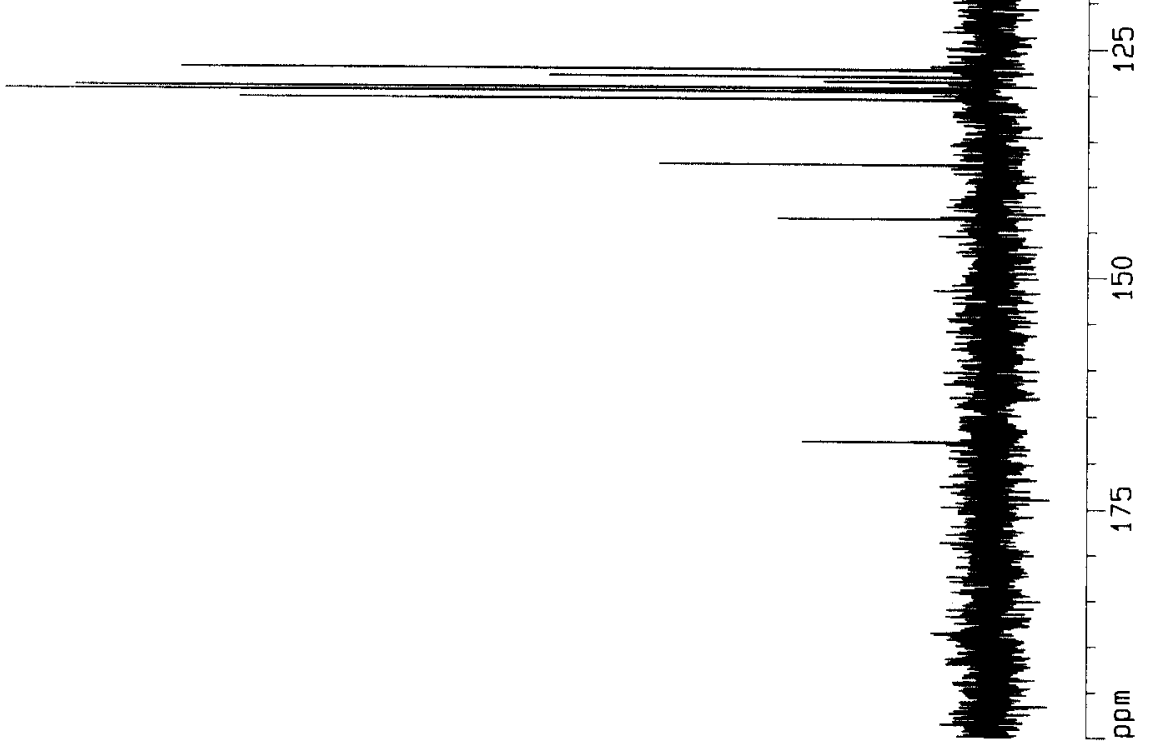

S-23 


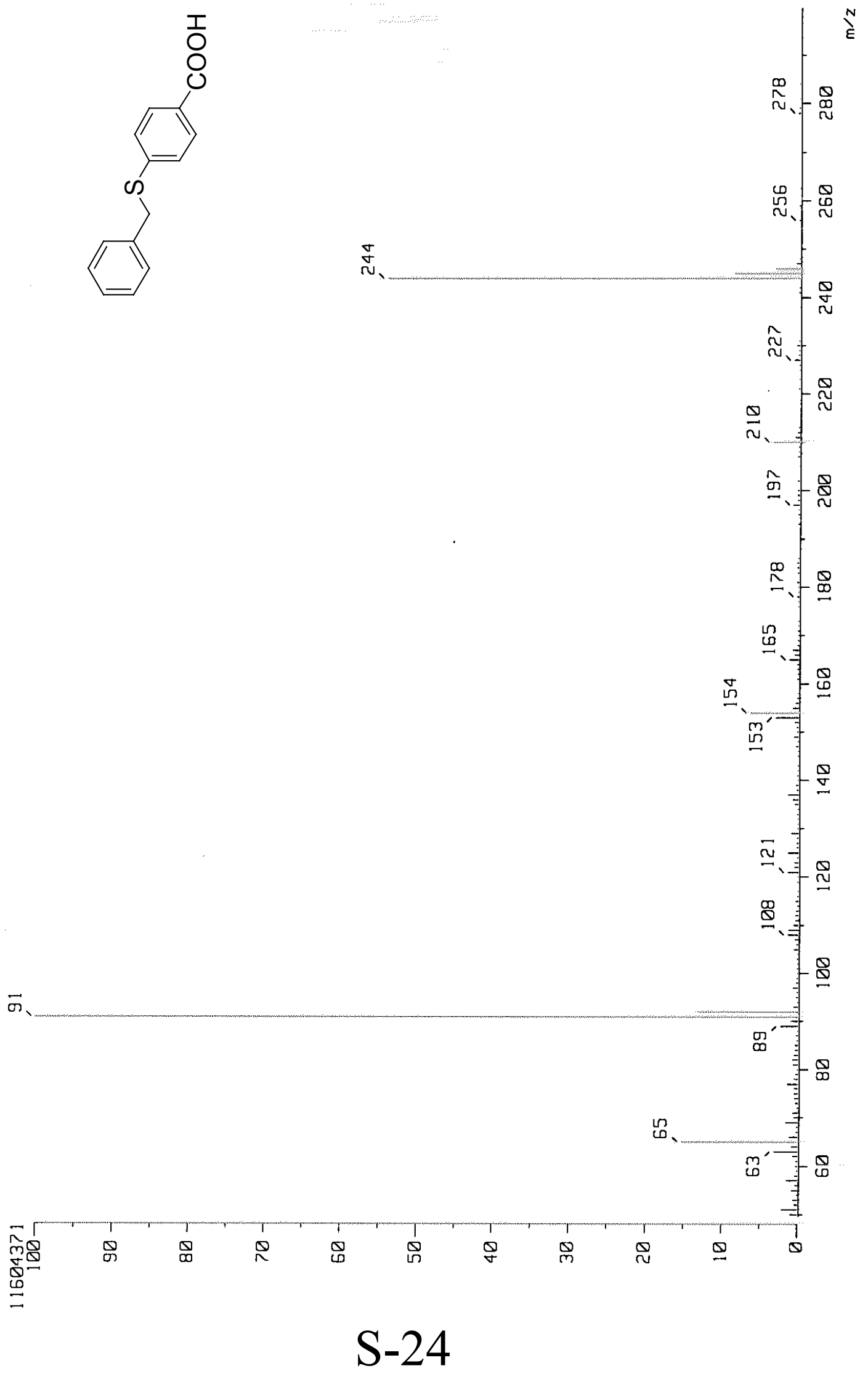



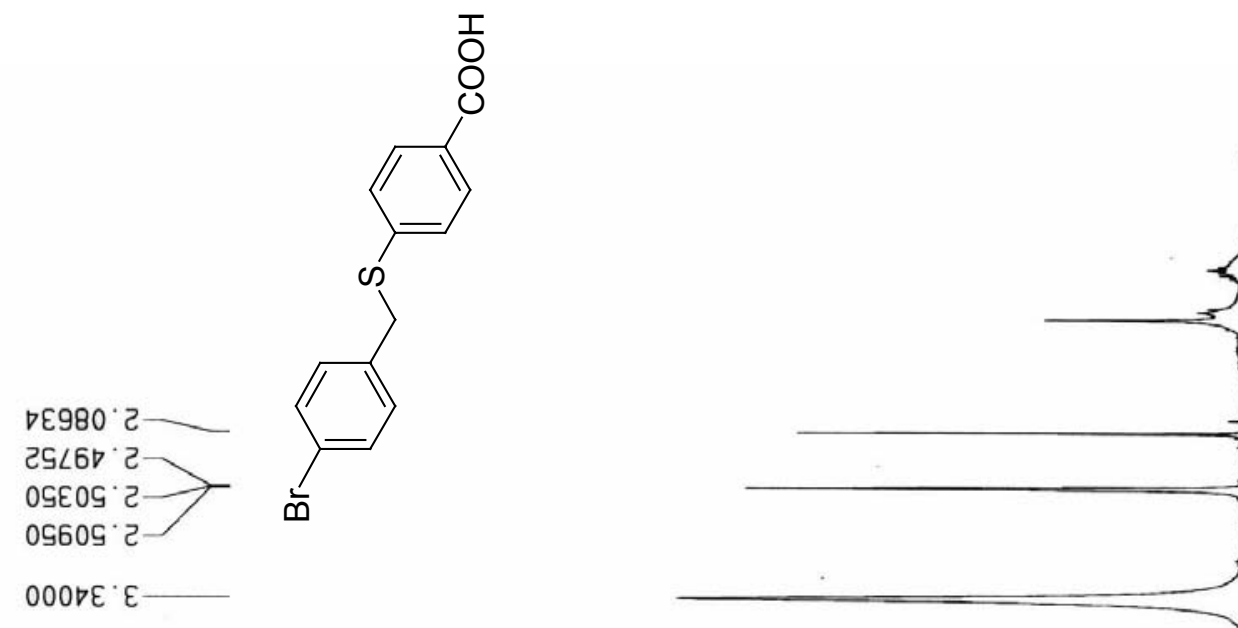

SEDEE

L695E '

EOSBE

9ट96E

ट9०ट०

6दा6०

096 IS $\angle$

SS $\angle 08$.

699E8 $\angle$

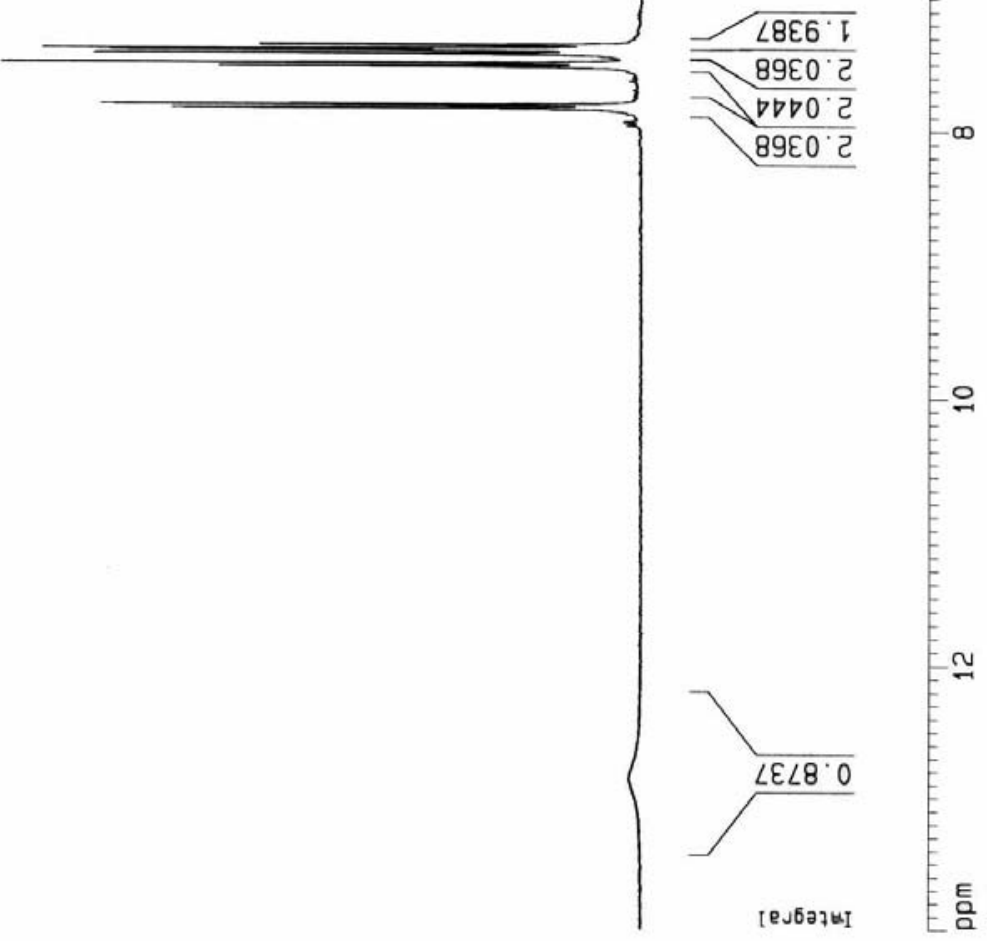

\section{S-25}




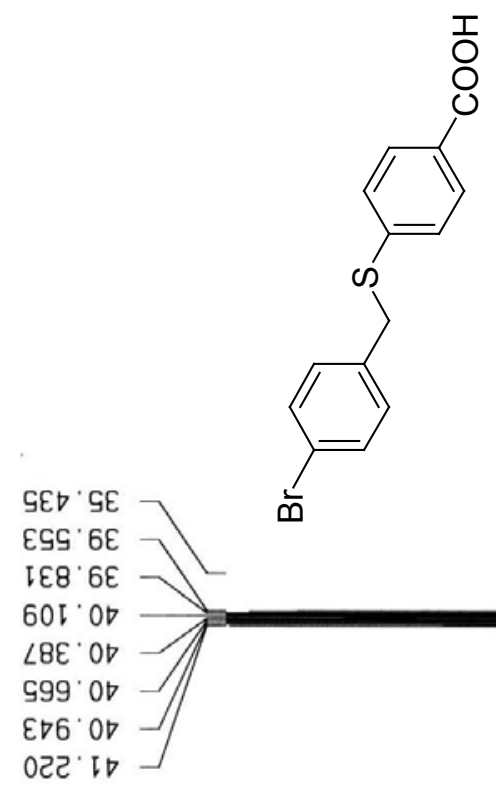

$\nabla \angle I \cdot L$

$\checkmark I G^{\circ} \angle 2 !$

$680^{\circ} 82 \mathrm{~T}$

$889^{\circ} 0 E I$

$\angle \angle 8^{\circ}$ IE

દLટ'ટદ I

LOD ' $\angle E I$

IIE $E \nabla$ I

$69 L^{\circ} \angle 9 \tau$ 


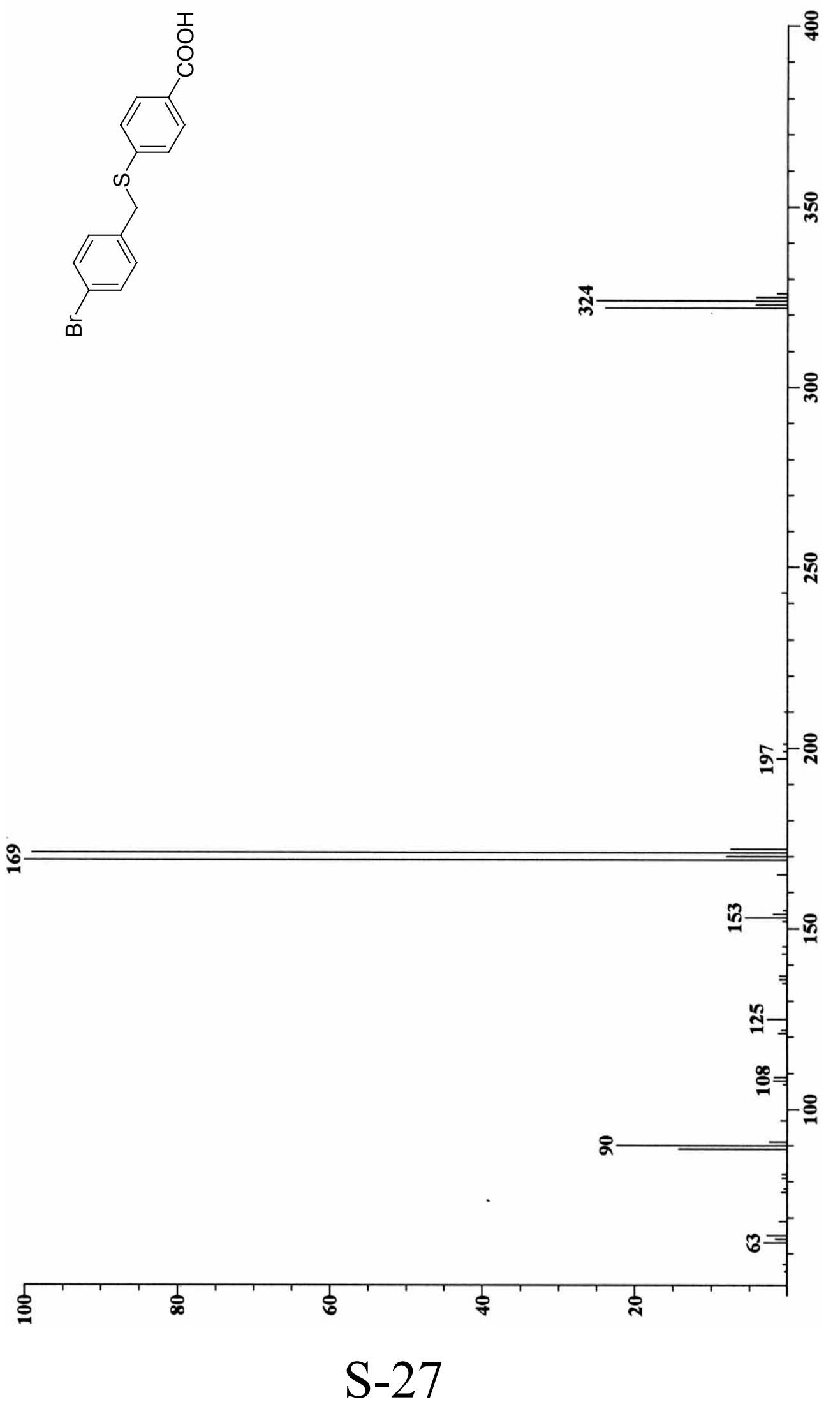




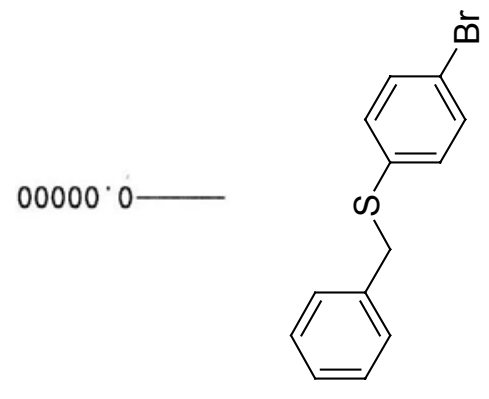

\section{$\varepsilon 己 \angle \nabla \mathcal{G}^{\circ} \downarrow$}
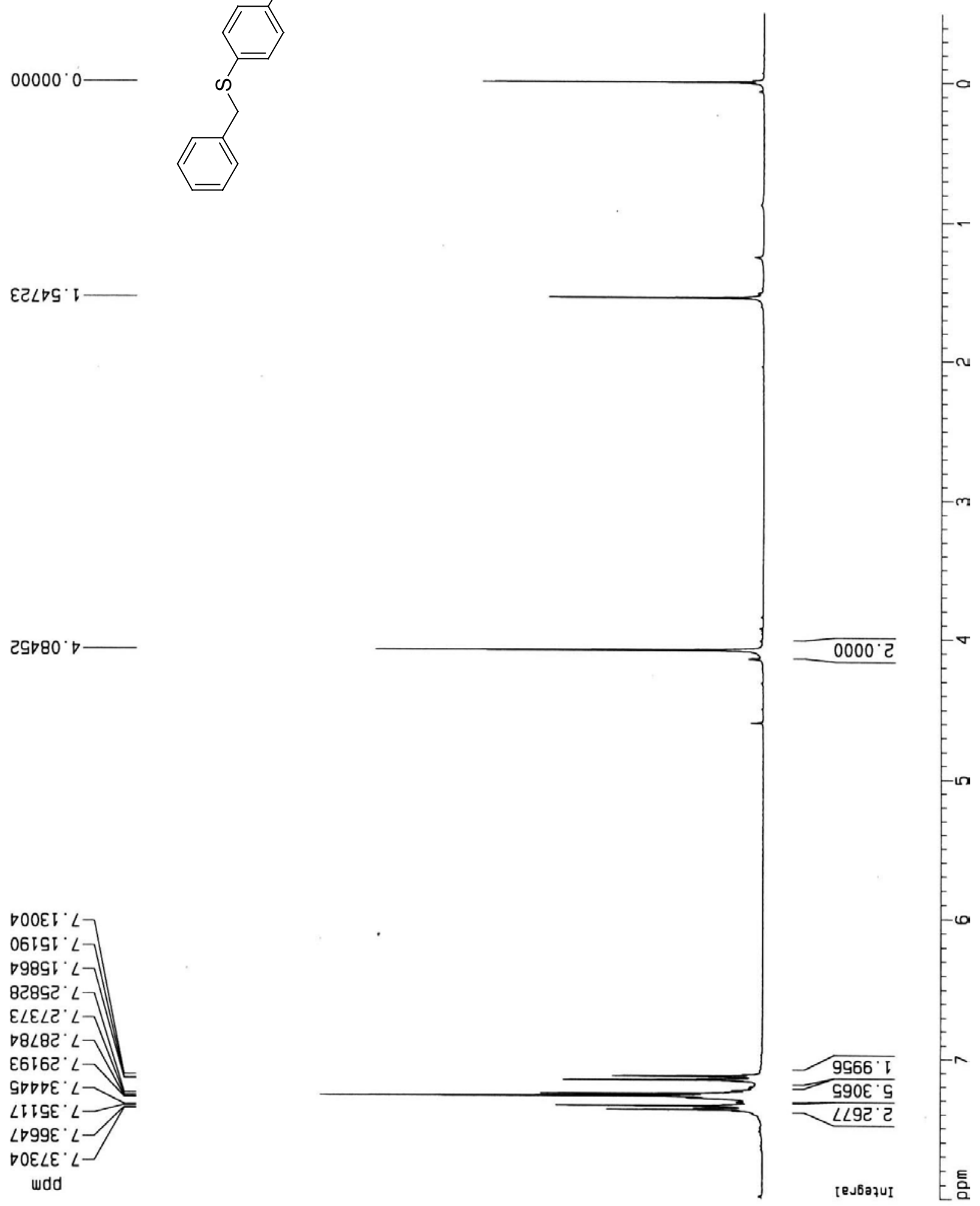


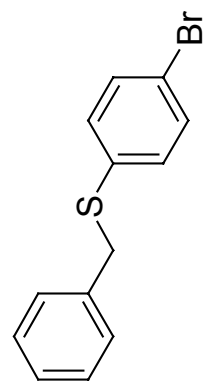

$66 \nabla^{\circ} 6 \varepsilon$

$066^{\circ} 9 L$

EIV $L L$

9E8 $\angle L$

6टL OCL

$82 L^{\circ} \angle 2 L$

096 8ट

เ8น '6ट

S68 IE I

6૬ટ ટદ

I L8 GEI

$6 ट \nabla^{\circ} \angle E \downarrow$ 


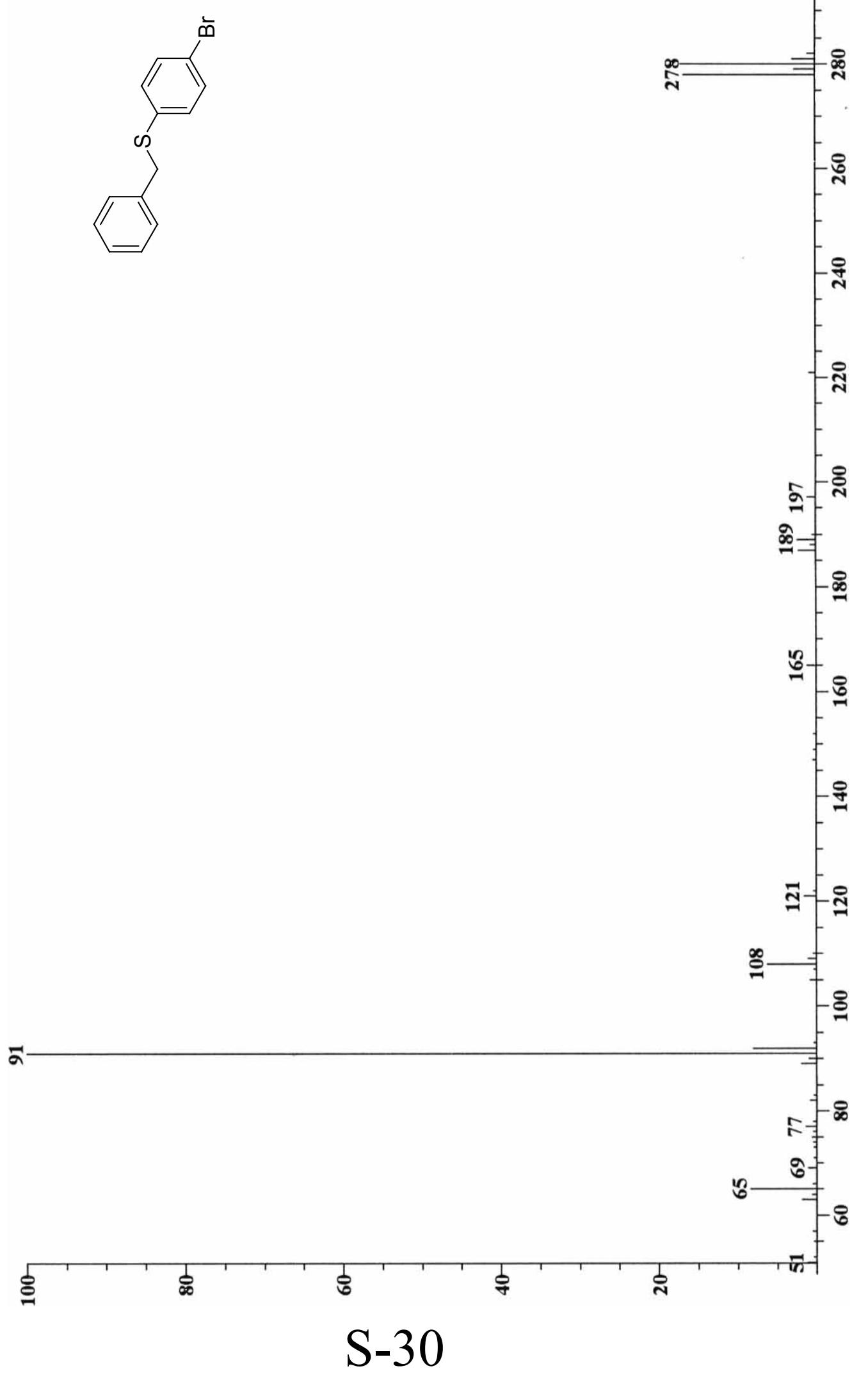




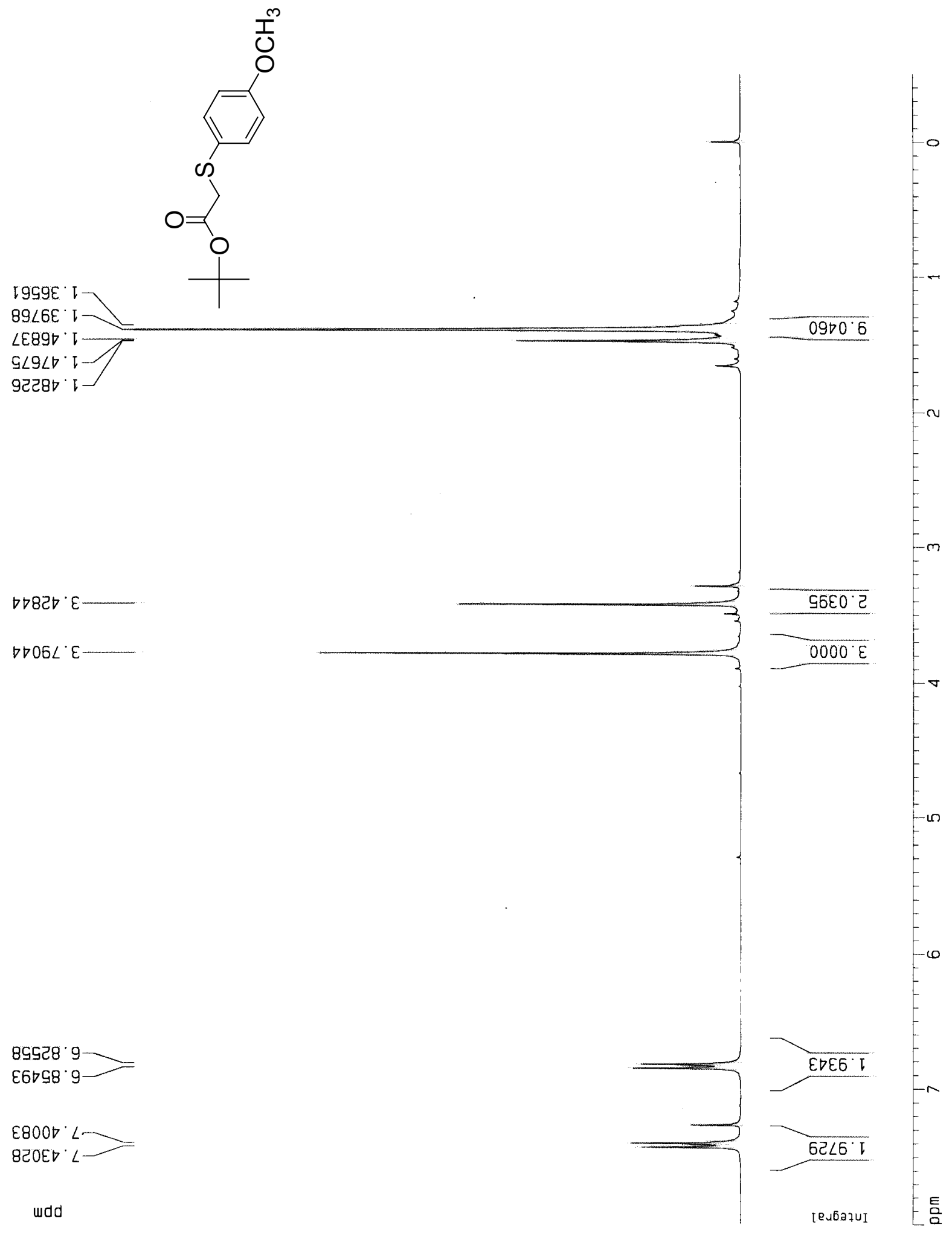

S-31 


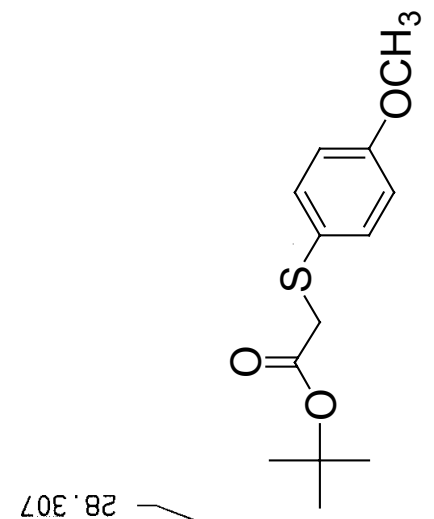

S6E 82

$900^{\circ} 07$

$92 L \cdot 59$

$\checkmark 10^{\circ} \angle L$

$8 E 0^{\circ} \angle L \longrightarrow$

$198^{\circ} \mathrm{LL}-$

$\varepsilon \angle 6^{\circ}$ โ8

टจ6॰ $จ$

$8 \nabla L \cdot 521$

$\varepsilon 己 E^{\circ} \triangleright E V$

$8 \angle 8^{\prime} 69 \tau$

$8 \nabla \nabla 69 \mathrm{I}$

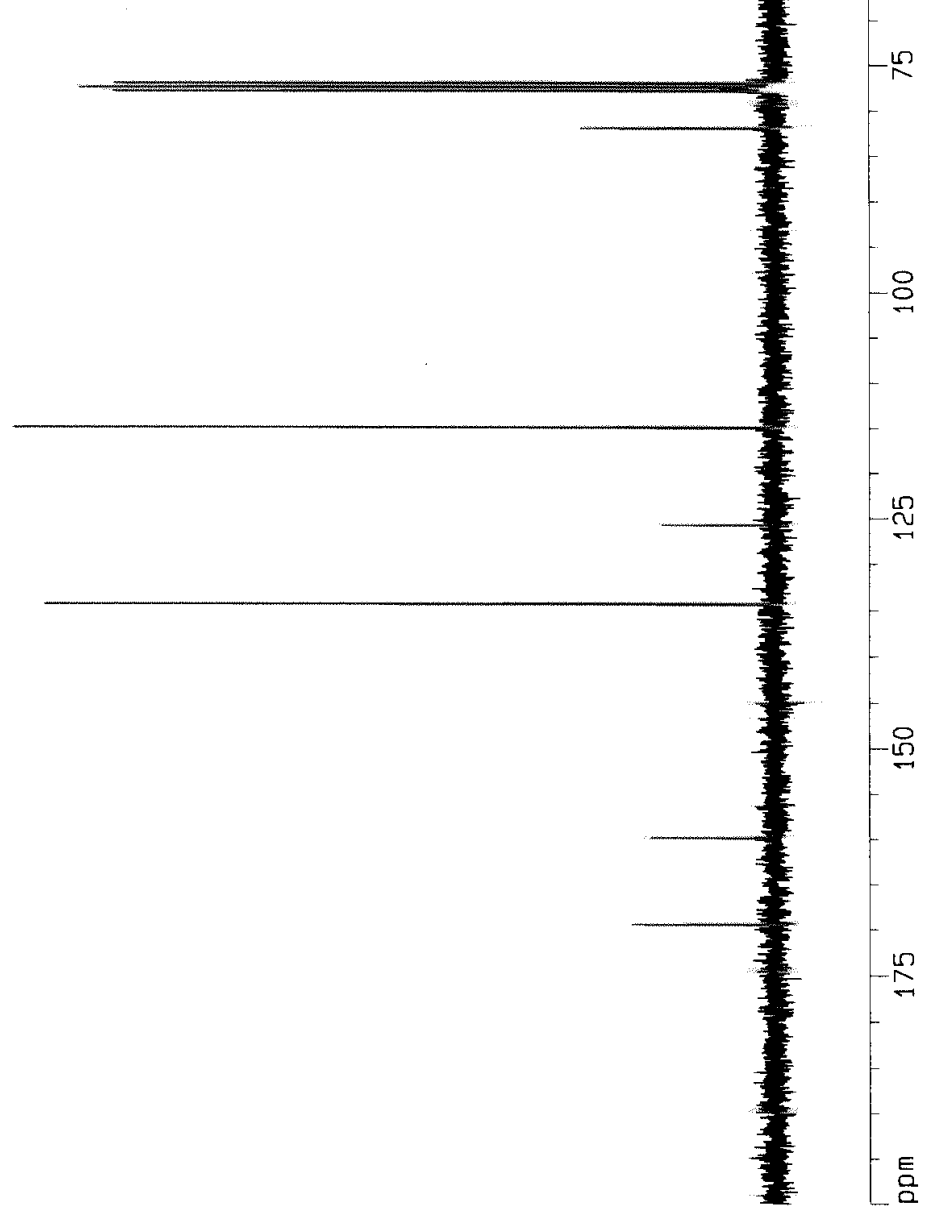

S-32 

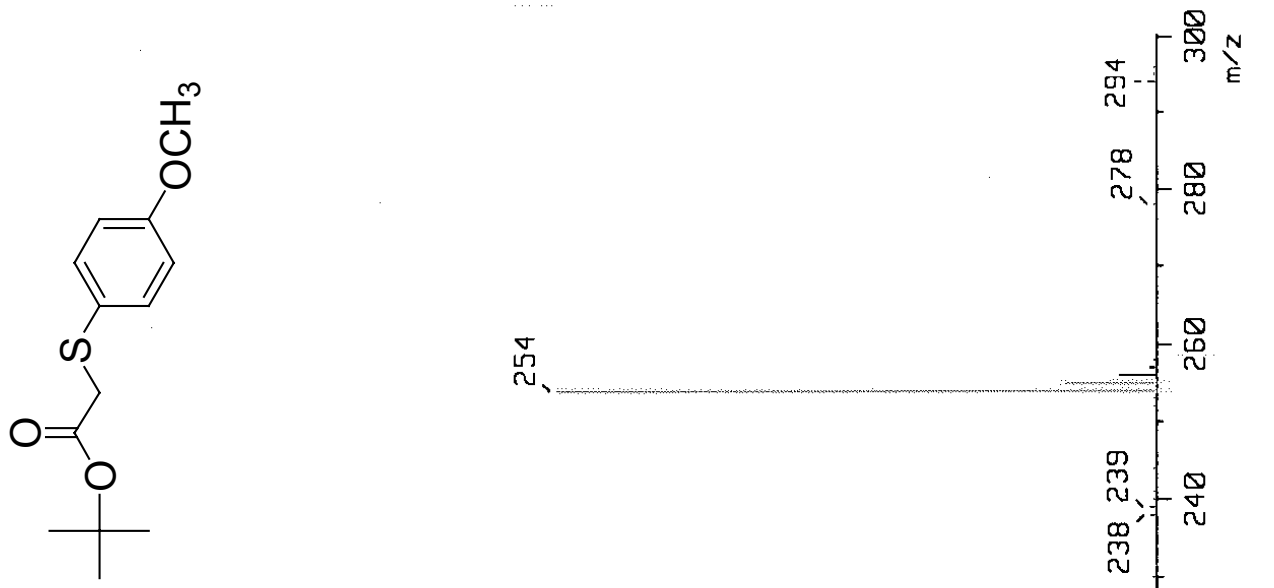

㽞

m.

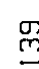

$\stackrel{0}{-}$

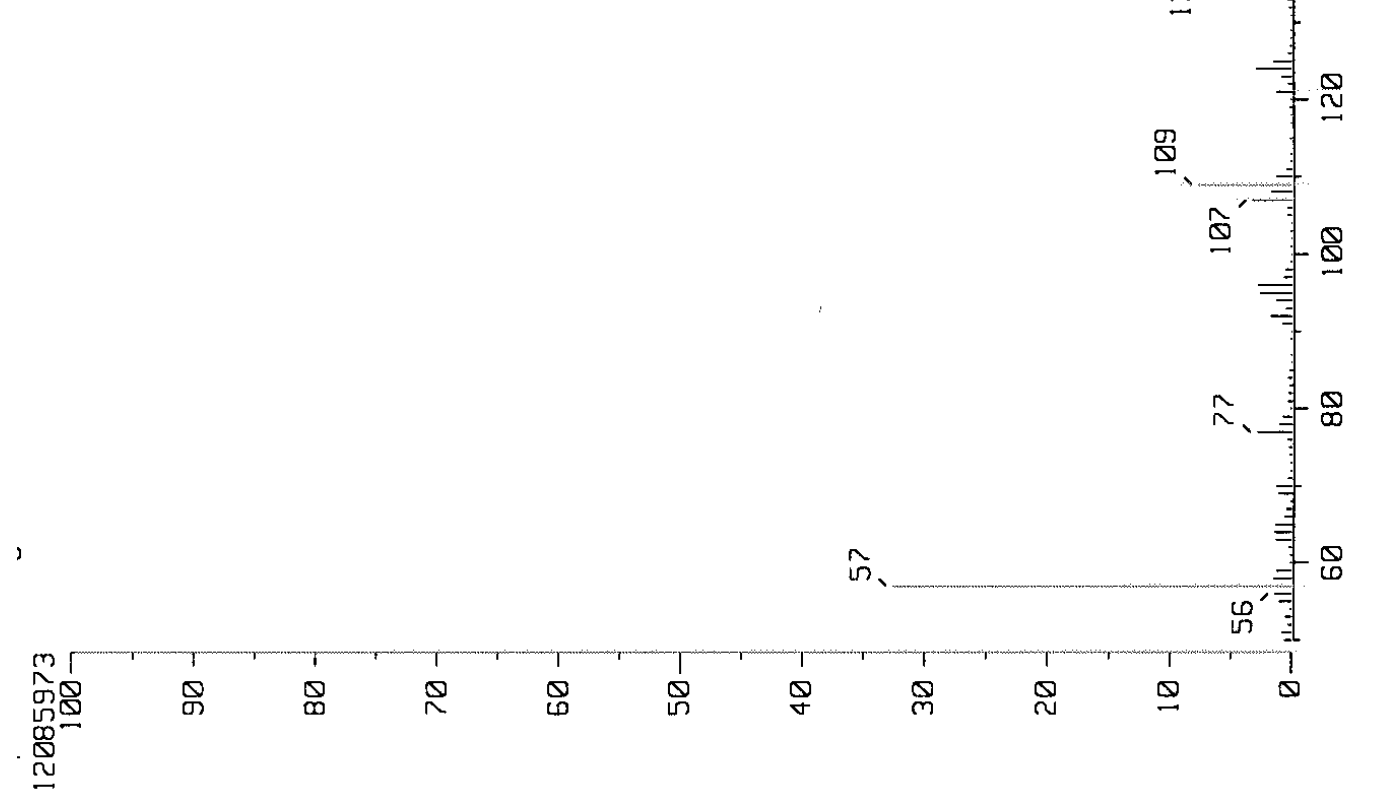

S-33 


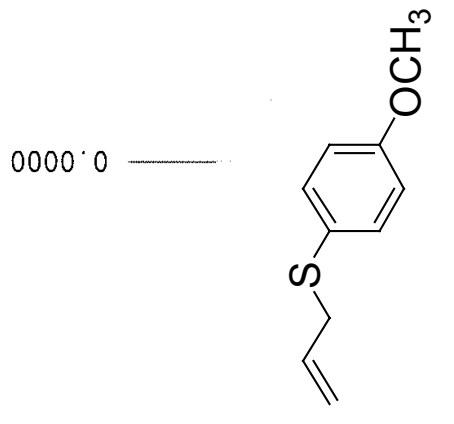

86ट๖

$\varepsilon \nabla \angle G^{\circ} \downarrow$

E692 ट

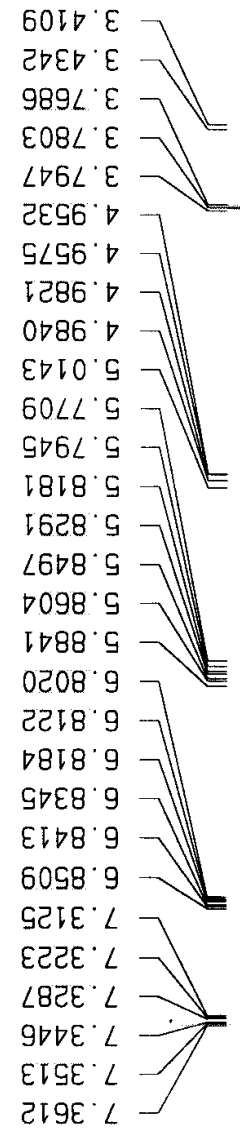

udd

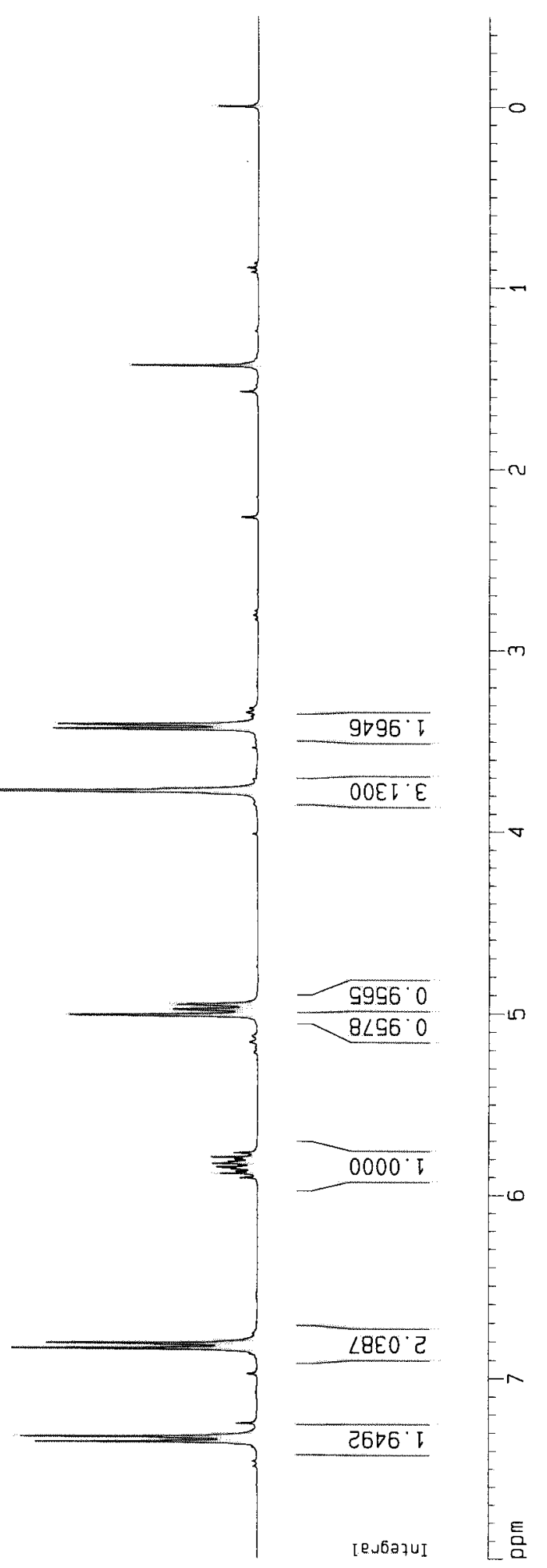




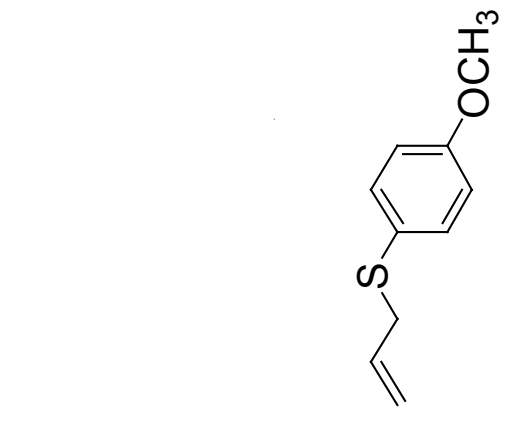

$\angle E L^{\prime} O E$

$\varepsilon \nabla L^{\prime} G E$

$\angle 69^{\circ}{ }^{\circ} G^{\prime}$

GEO ' $\angle L$

$69 \nabla^{\prime} \angle L$

288. $\angle L$

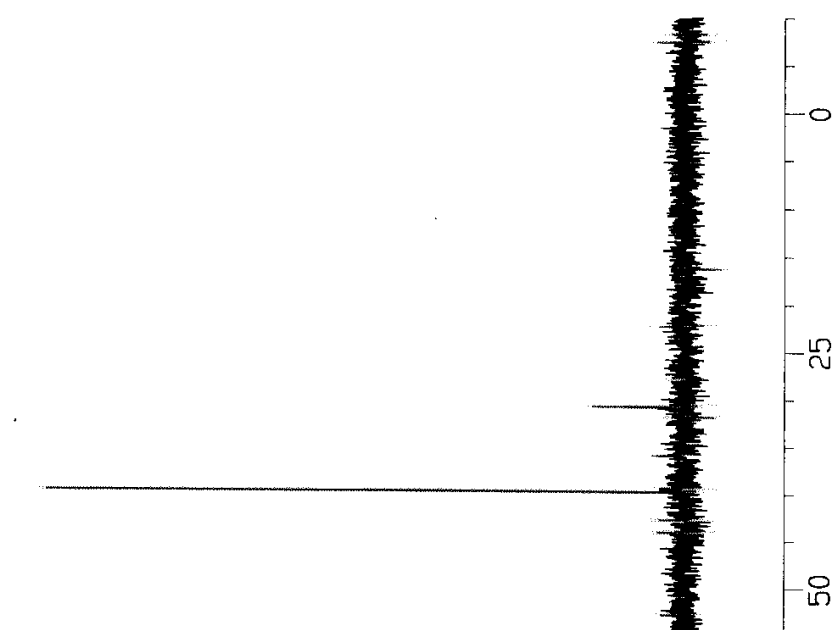

윰

Gट8 $\triangle 1$

$299^{\circ} \angle I I-$

ट๐ट.9टा

OLE $\nabla E \downarrow$

IG० $จ E \downarrow$

$96 \nabla^{\circ} 69 \tau$

S-35 

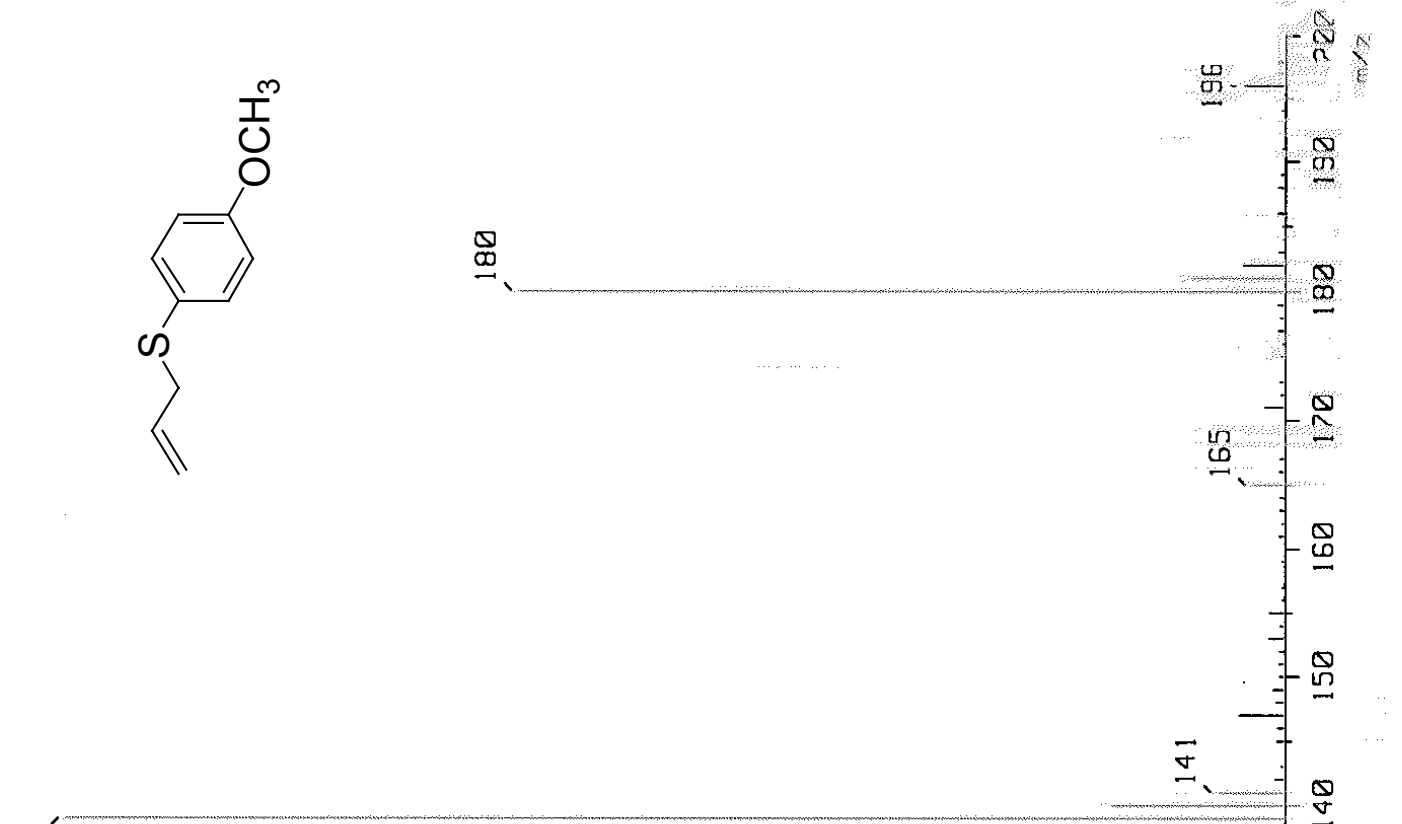

$m$
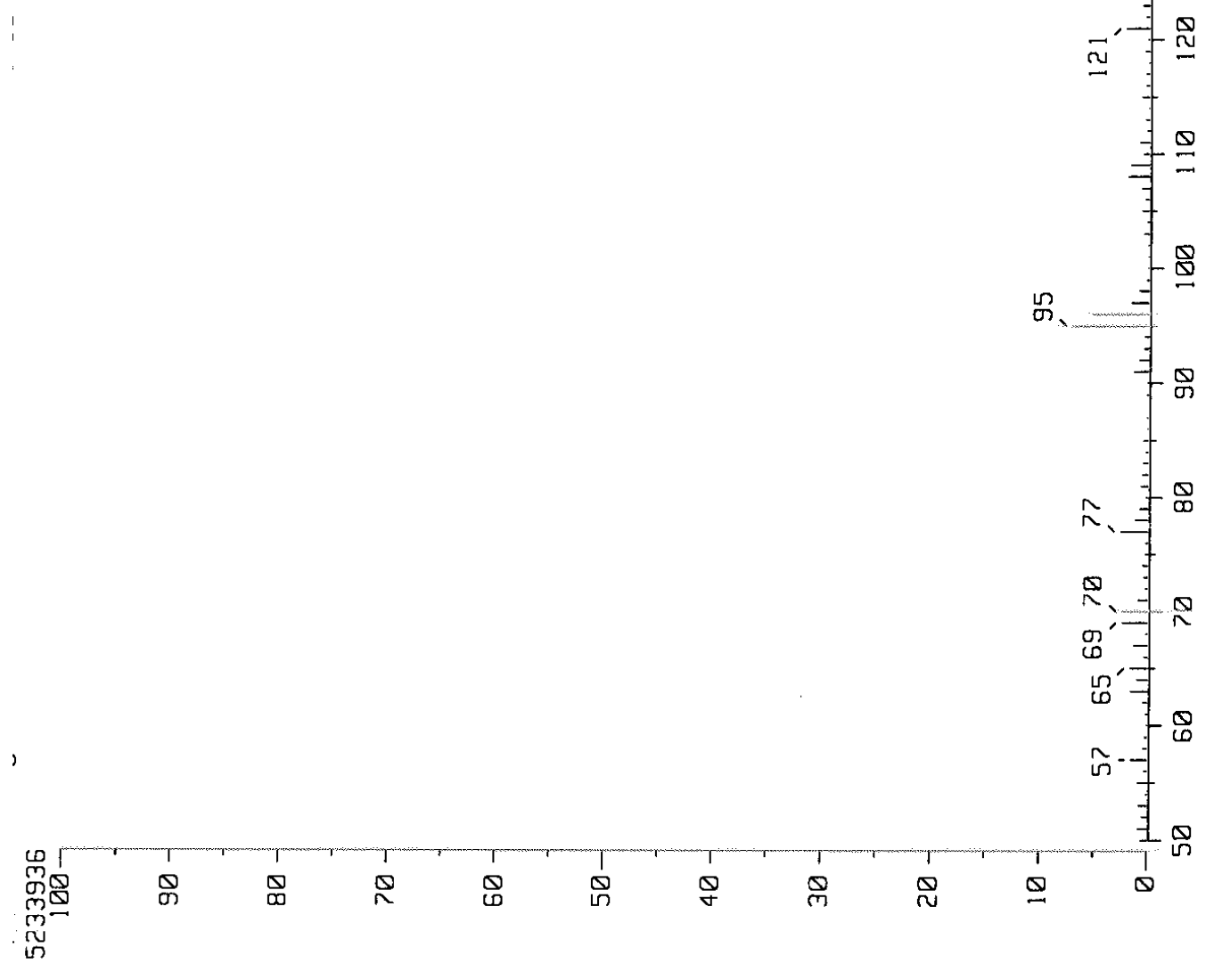

S-36 


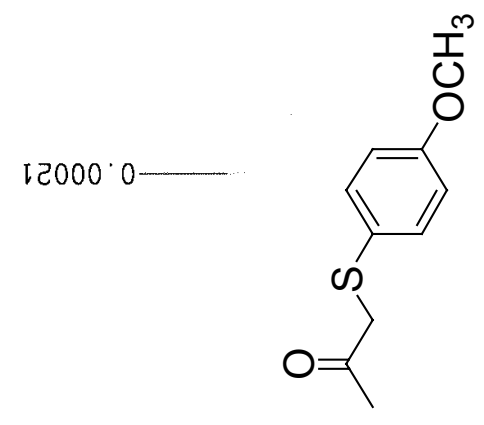

$\angle 9689^{\circ} \downarrow$

EL8Sट'ટ-

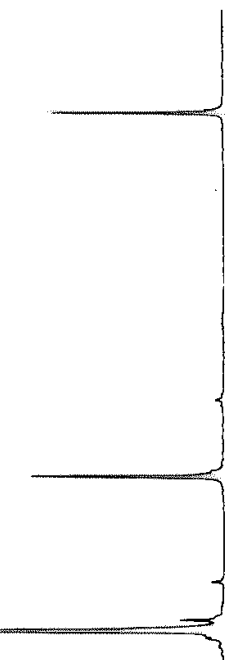

$\nabla \varepsilon \angle \nabla \mathcal{G}^{\circ} \varepsilon$

OS88L' $E$

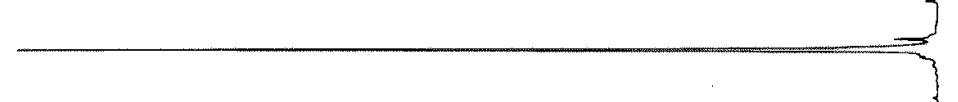

$8 E 10^{\circ} \varepsilon$

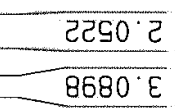

\section{$29928^{\circ} 9$}

$98 \nabla 98^{\circ} 9$

$\nabla \angle E E E^{\prime} \angle$

BOE9E.

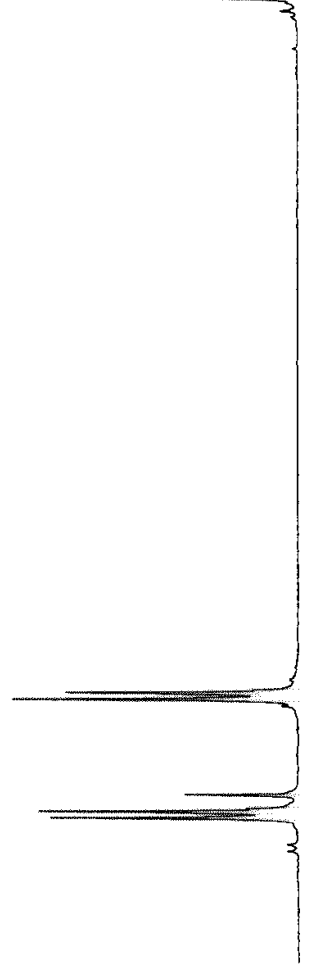

wdd
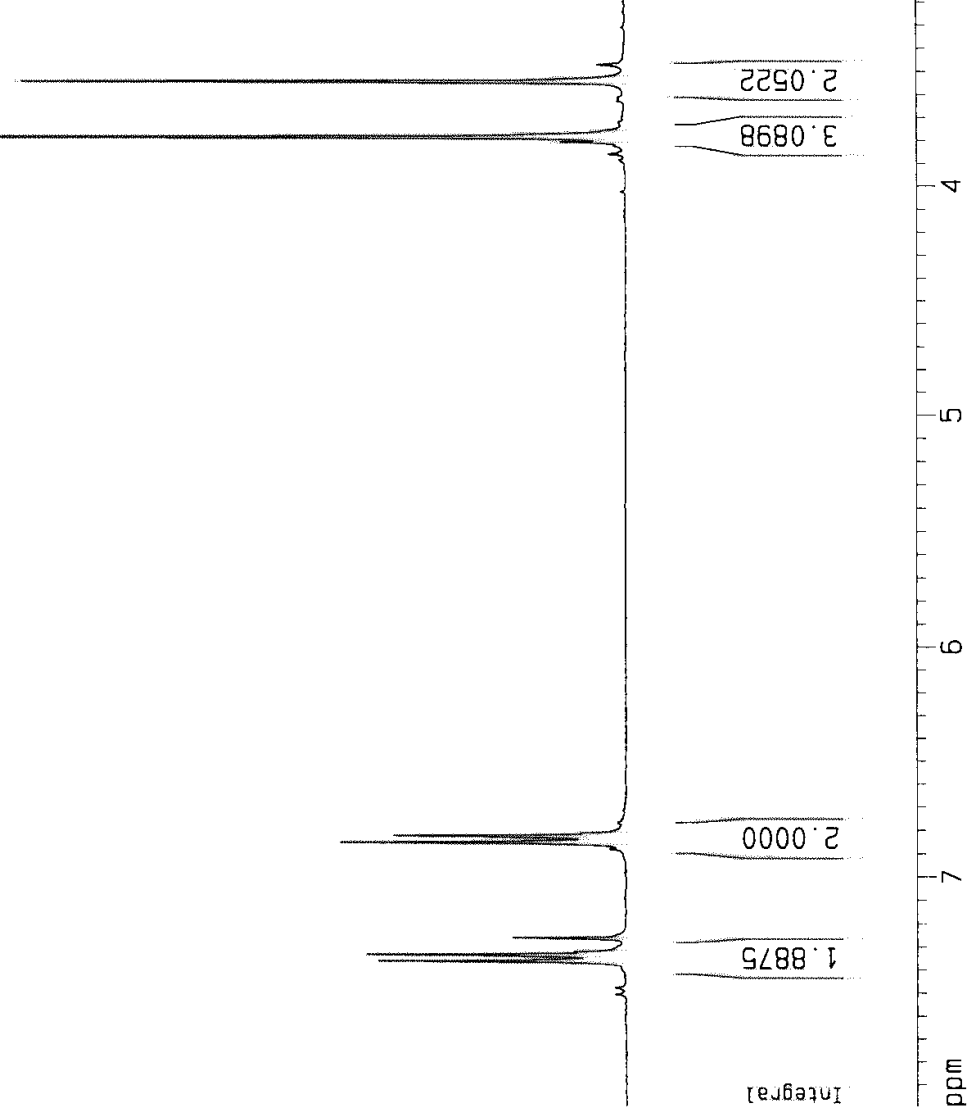


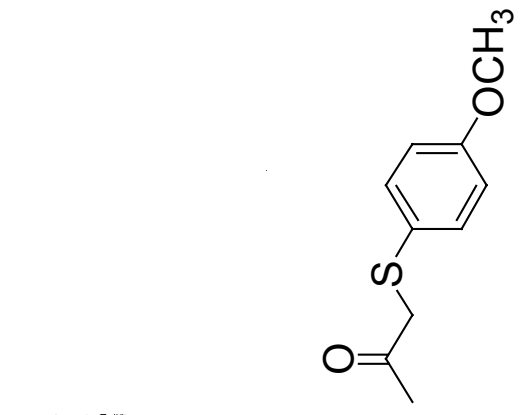

$990^{\circ} 82$

$096^{\prime} 90$

$\angle 2 L G G$

$966 \cdot 9 L$

6IV' $\angle L$

$2 \nabla 8^{\circ} \angle L$

602515

$086^{\circ} \nabla 2 T$

$0 \subseteq 0^{\circ} \nabla E \downarrow$

$\angle 66^{\circ} 691$

$\angle 66^{\prime} \varepsilon 0 C^{\prime}$

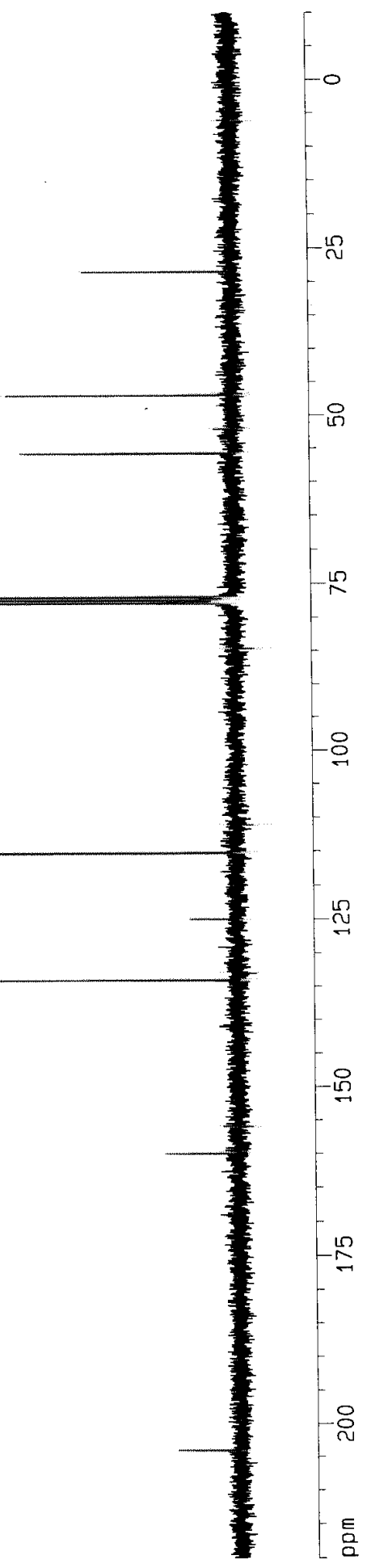

S-38 
$\stackrel{\mathscr{N}}{\sim}$

m

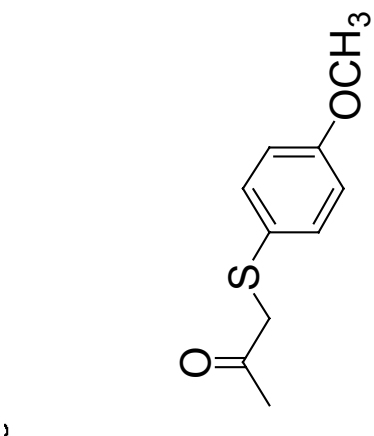

!

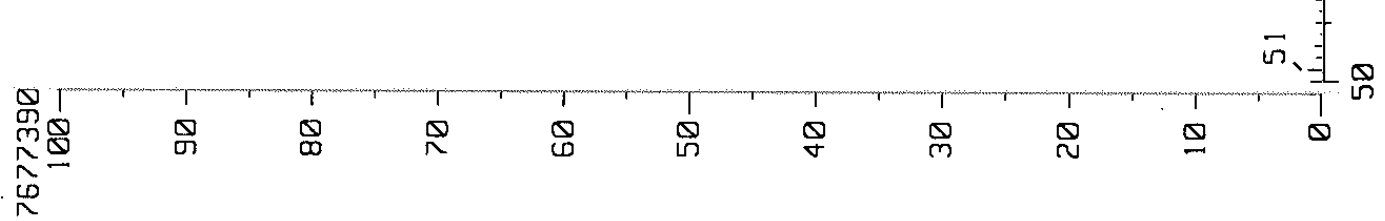

S-39 


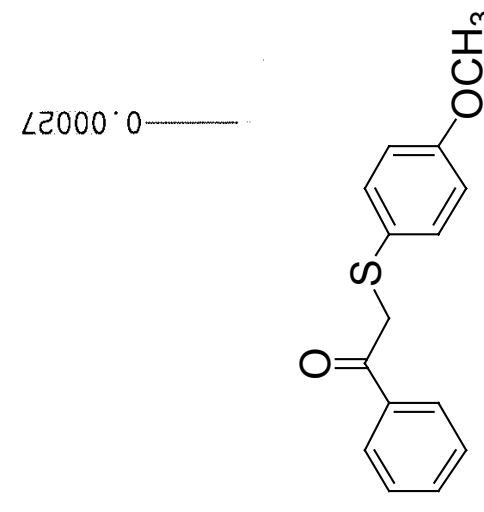

$9 \angle 09 L^{\circ} \varepsilon-$

ट६9 $\angle L ' \varepsilon-$

S88I' 0
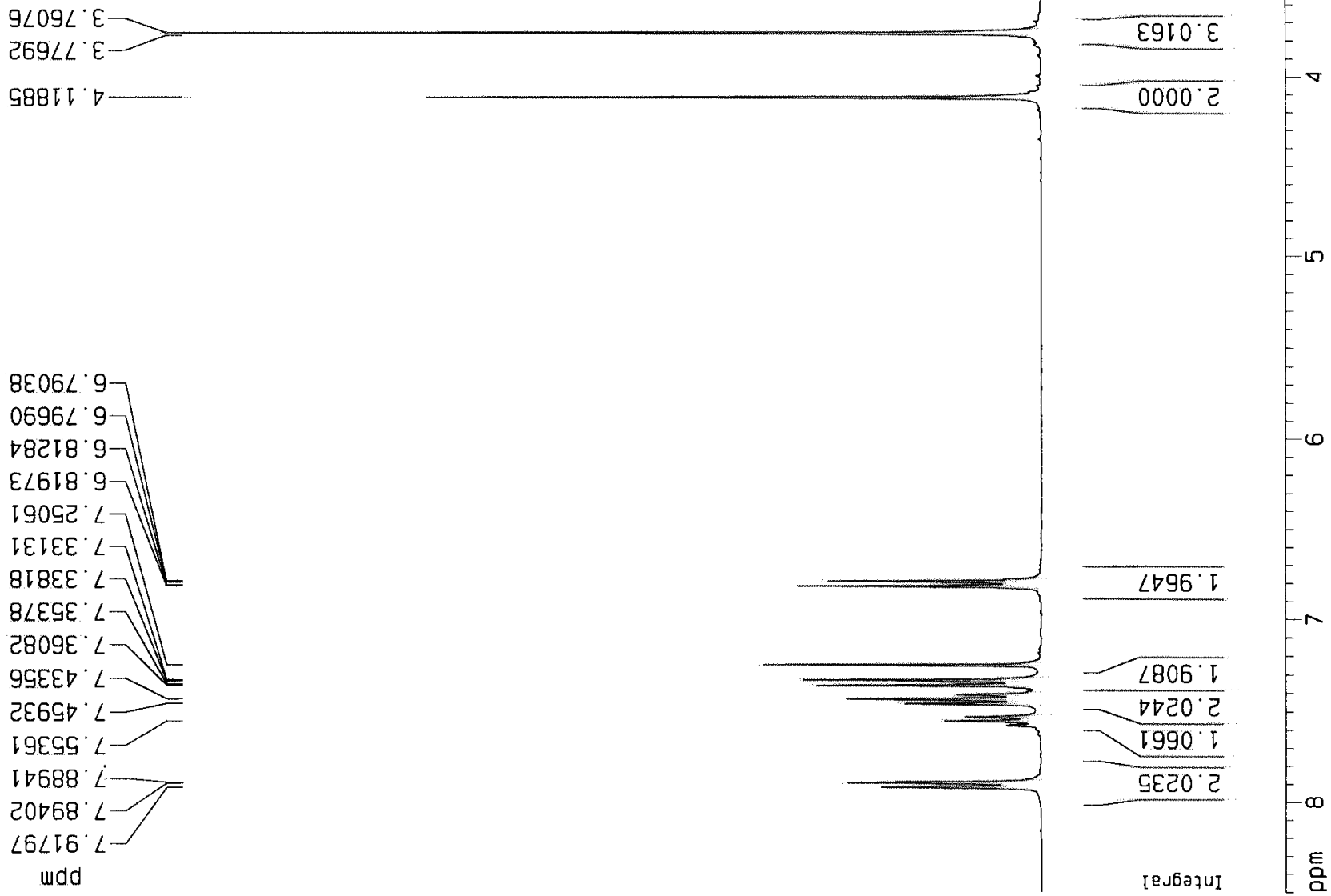


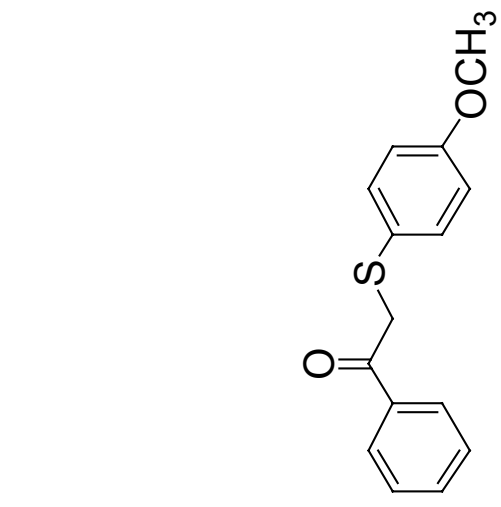

$\angle O Z^{\circ} \varepsilon \nabla$

$61<59$

$080^{\circ} \angle L$

EOS $\angle L$

SOL' $L L$

$\angle 26^{\circ} \angle L$

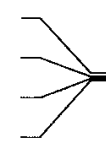

IDI gTt

E20 9टा 0म0 6टा

9EI 6己厂

$82 \angle$ EE

6E0 $9 E$ ?

$\angle L G^{\circ}$ GE T

$99 \tau^{\circ} 09$

$62 L \vee \nabla 6 I$
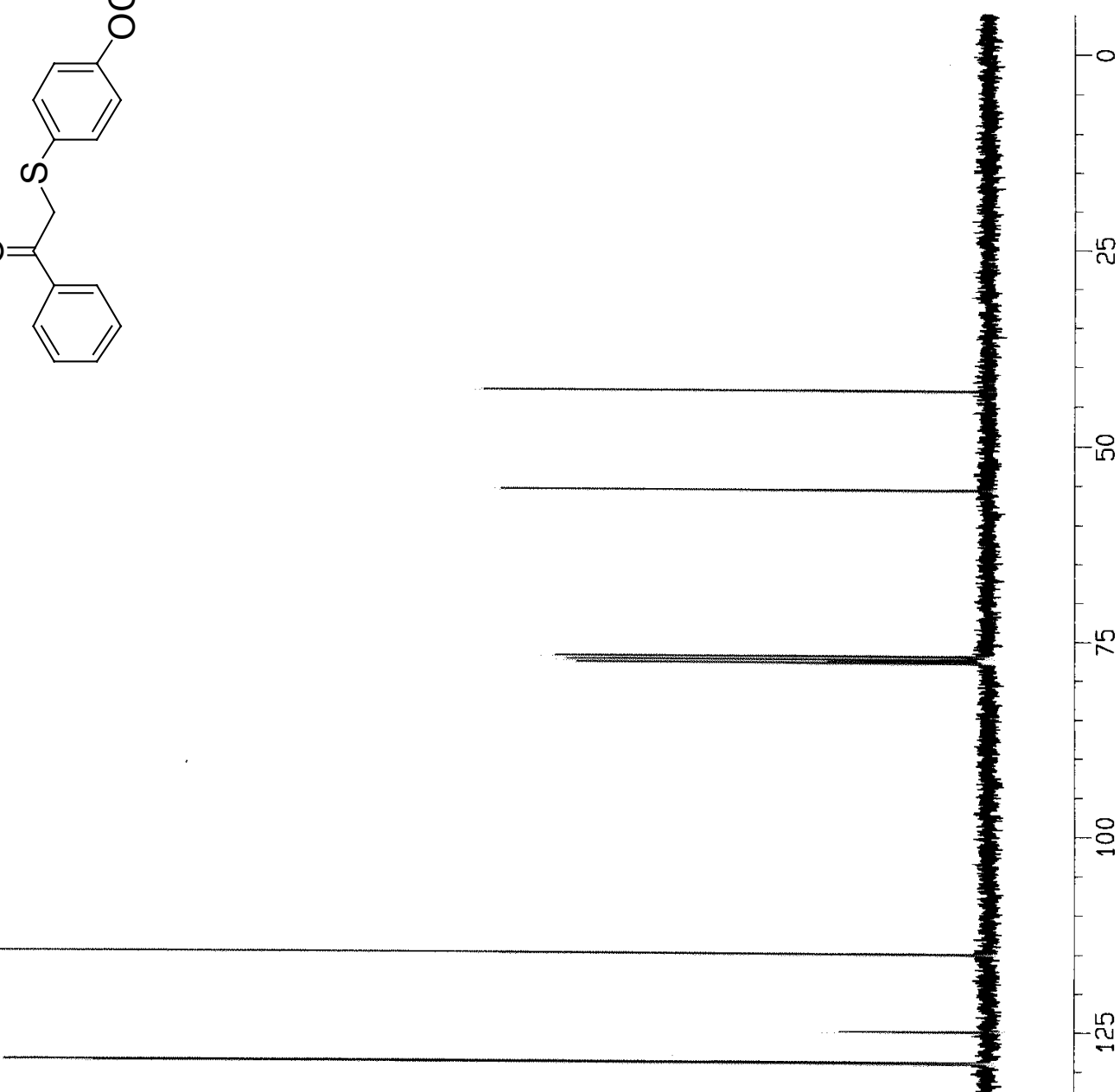


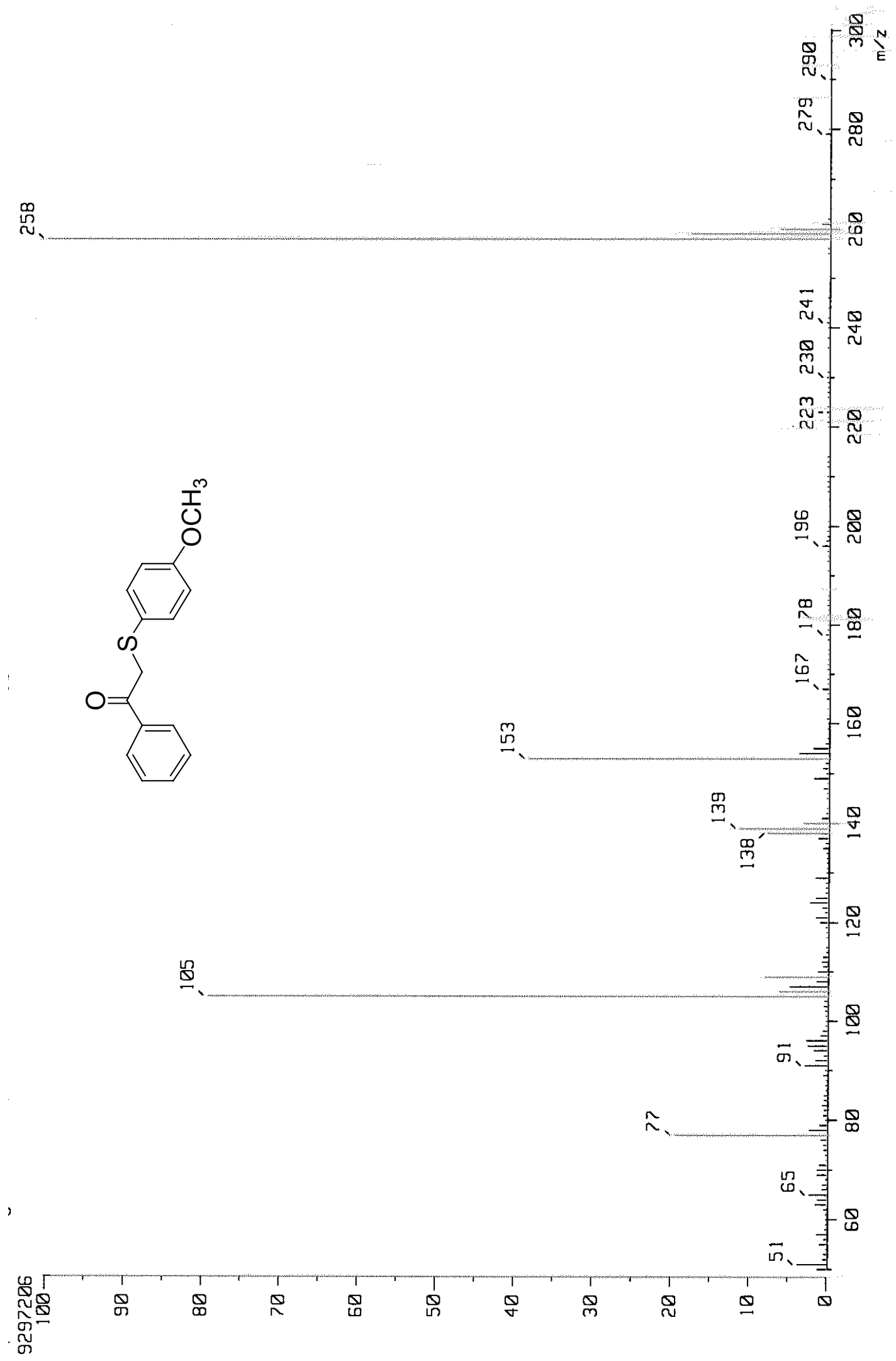

S-42 
$00000^{\circ} 0$

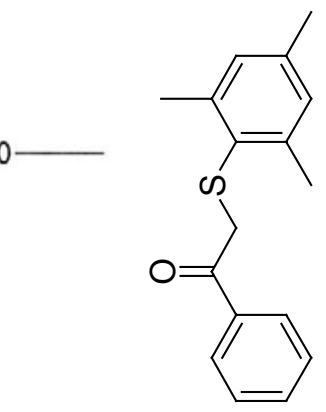

98699' โ

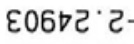

SOLLC

S06 $\angle E \cdot 己$

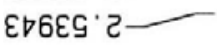
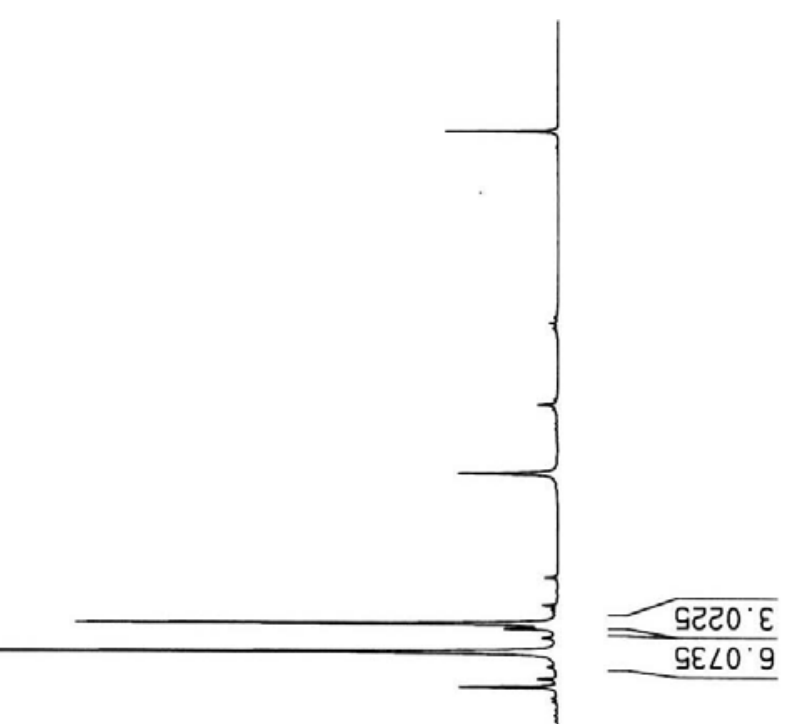

DELट6 ${ }^{\circ} \varepsilon$

$68060^{\circ}$
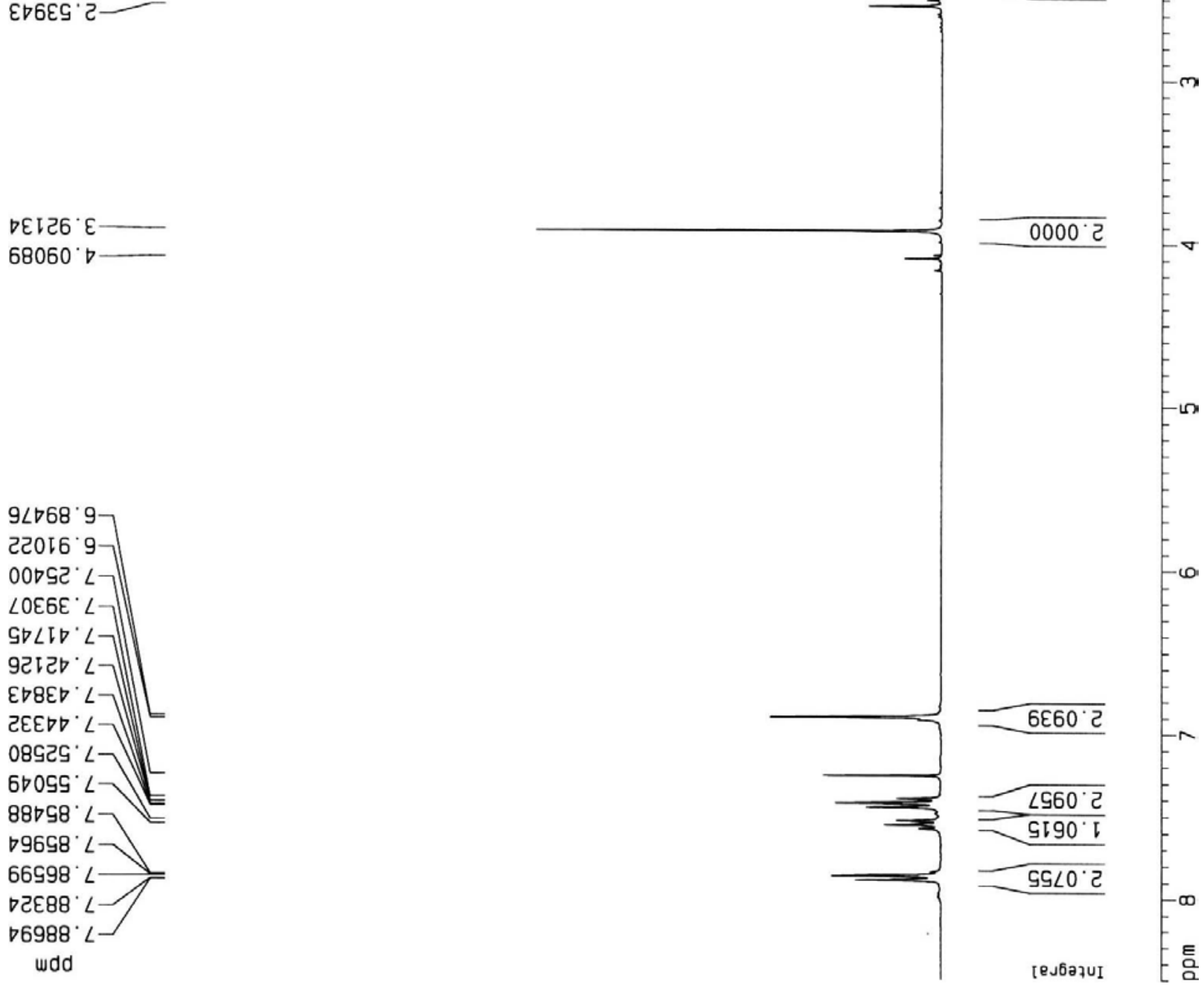
SOt. IT $960^{\circ}$ ट己

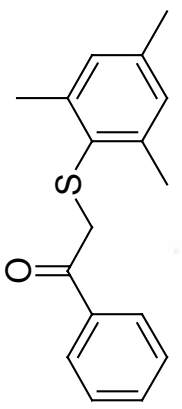

BLC I

$286^{\circ} 9 L$

SOD $\angle L$

$628^{\circ} \angle L$

EE6 ' 8ट I

$160^{\circ} 621$

$\angle 99^{\circ} 62$

ट09 $\varepsilon E$

टLट $6 E$

$002^{\circ} \mathrm{CD}$

$9 \angle G^{\circ} \varepsilon \nabla \downarrow$

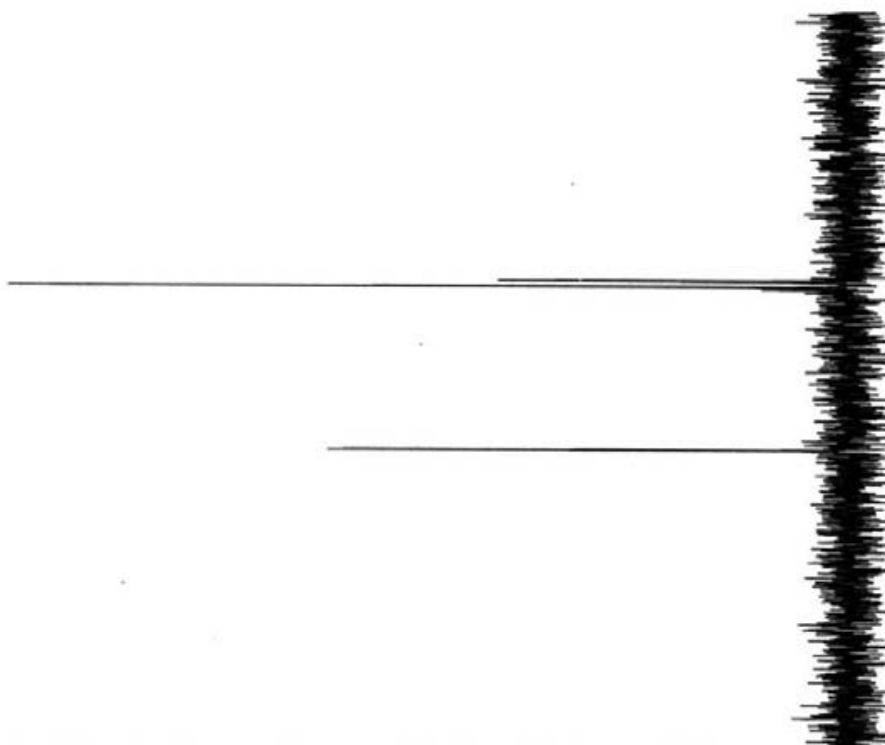

$868^{\circ} \vee 6 \downarrow$ 


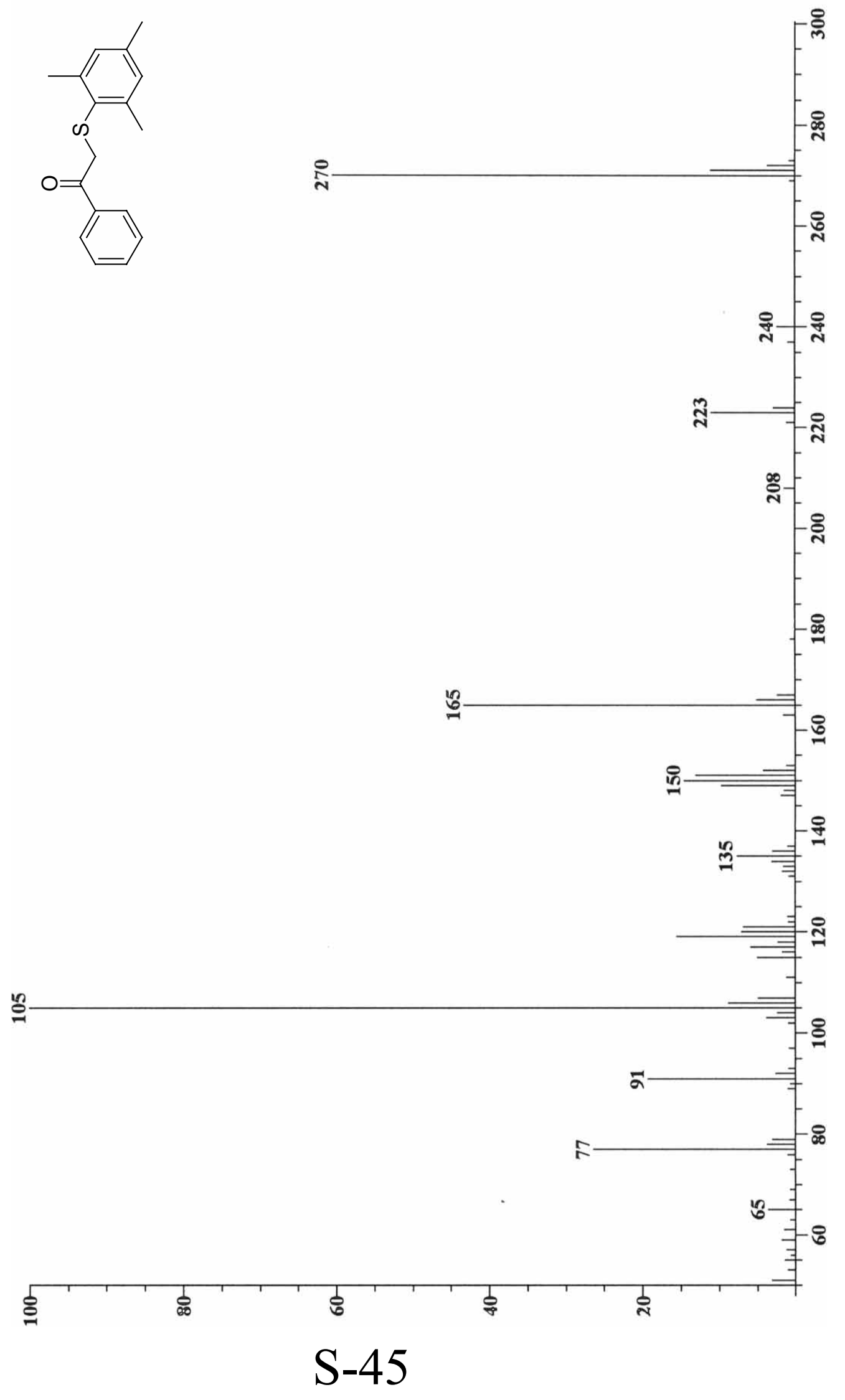




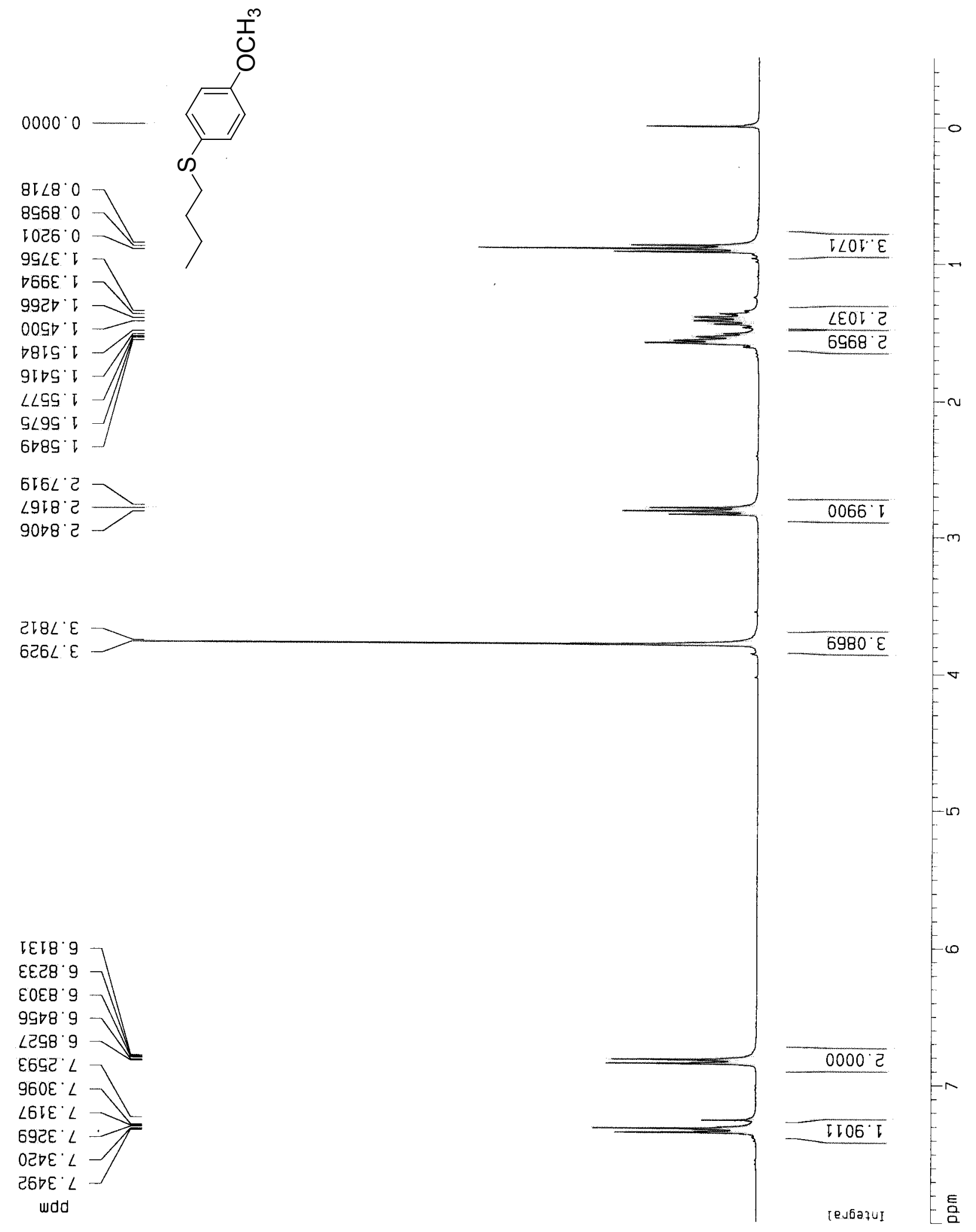



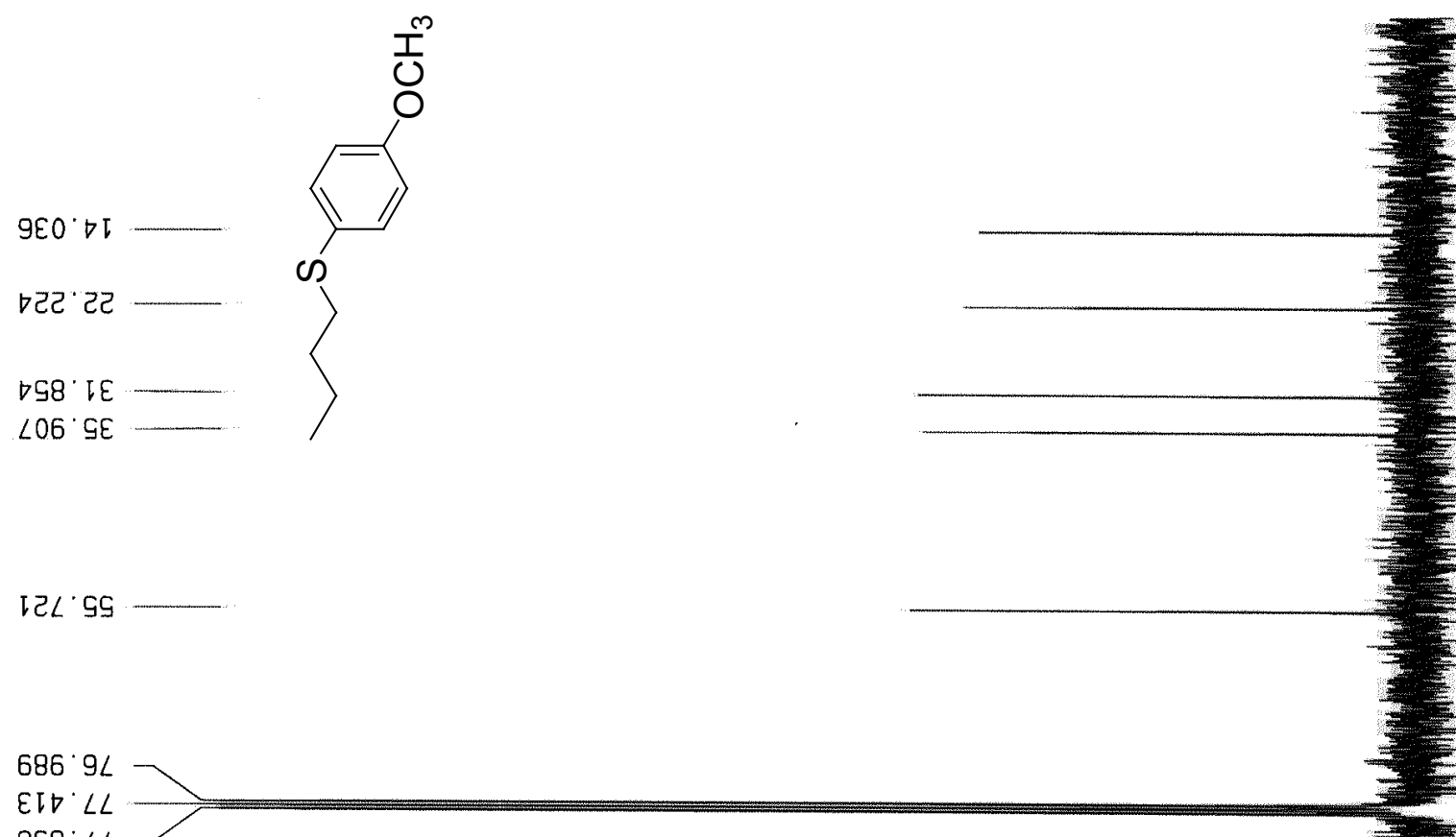

$686^{\prime} 9$

EIt $\angle L$ GEQ $\angle L$

$688 \cdot \downarrow \downarrow$

$G \angle E^{\prime} \angle Z \downarrow$

86ट EE

$\angle I T 65 I$ 


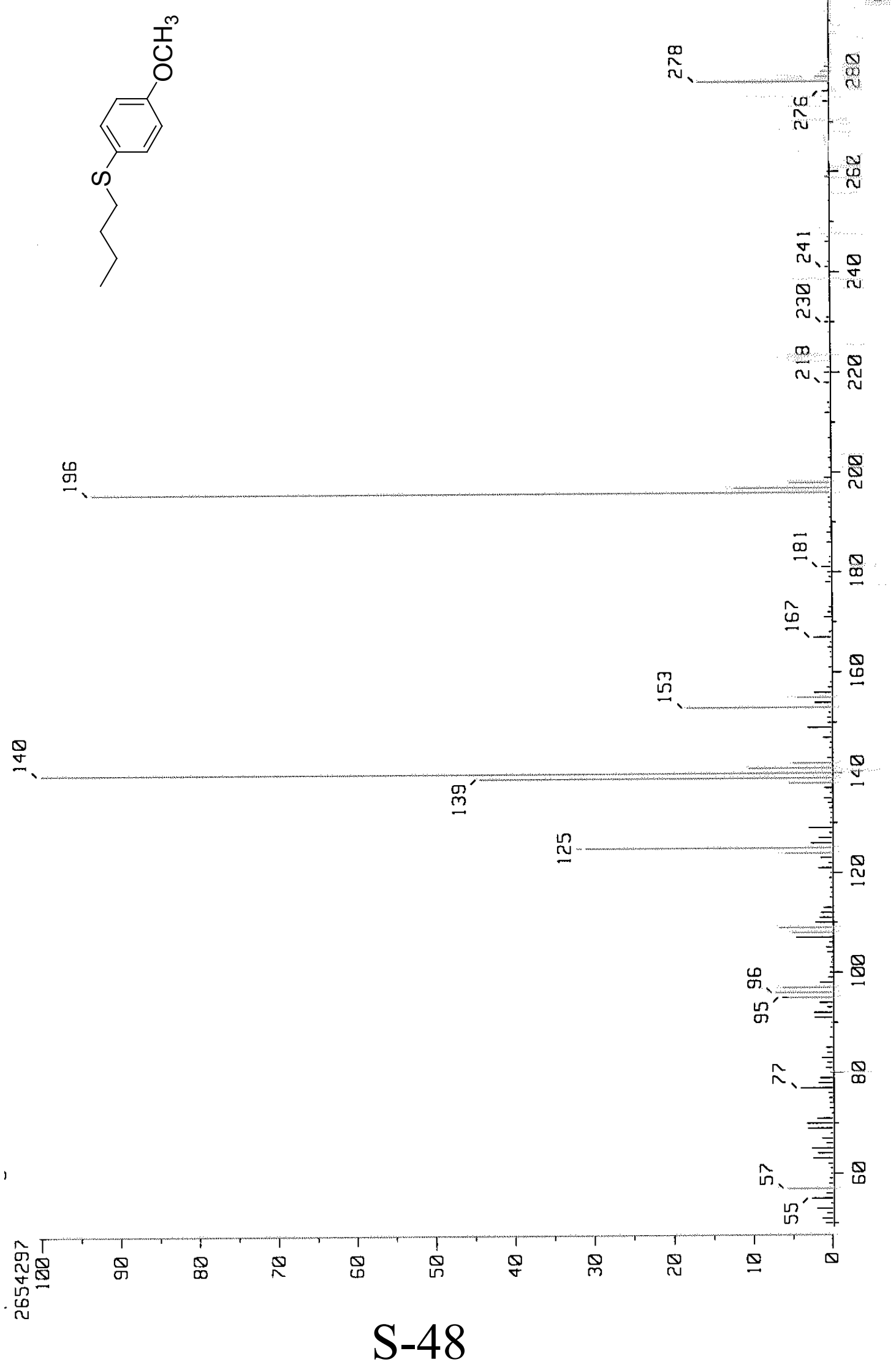


$27800^{\circ} 0$

$\angle C 610^{\circ} 0$

S2589.

Sट280 2

$\varepsilon 9 \angle 60^{\circ} \mathrm{C}$

9E9OT' 2

89ाटा'ट

9टाEI

SL9VI' 2

टEEDट $\varepsilon$

6ट89ट $\varepsilon$

$\angle G \angle 6 C^{\circ} \varepsilon$

$\mathcal{G} \angle 8 \angle 8^{\circ} \varepsilon$

$56006^{\circ} \varepsilon$

$99806^{\circ} \varepsilon$

BISI6 $\varepsilon$

$0 \angle \nabla C \sigma^{\circ} \varepsilon$

$\angle \varepsilon 89 \sigma^{\circ} \varepsilon$

$\angle 66966^{\circ} E$

OSEO

टOEIO

OS610 0

$60 \angle 20 \circ$

ट5600 $\circ$

I66E0 9

$91590^{\circ} 9$

$\nabla \nabla 0 \angle 0^{\circ} \mathrm{S}$

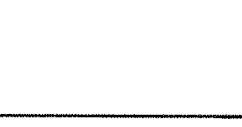

$\sqrt{2+20}$

1

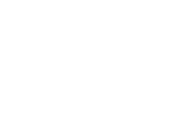

政

6Egा L $L$

๖G颔 $L$

SDSDI' $\angle$

$\angle G I \angle L \angle Y$

ตั

$8 \pi \mathrm{ICO}^{\circ} \angle \longrightarrow$
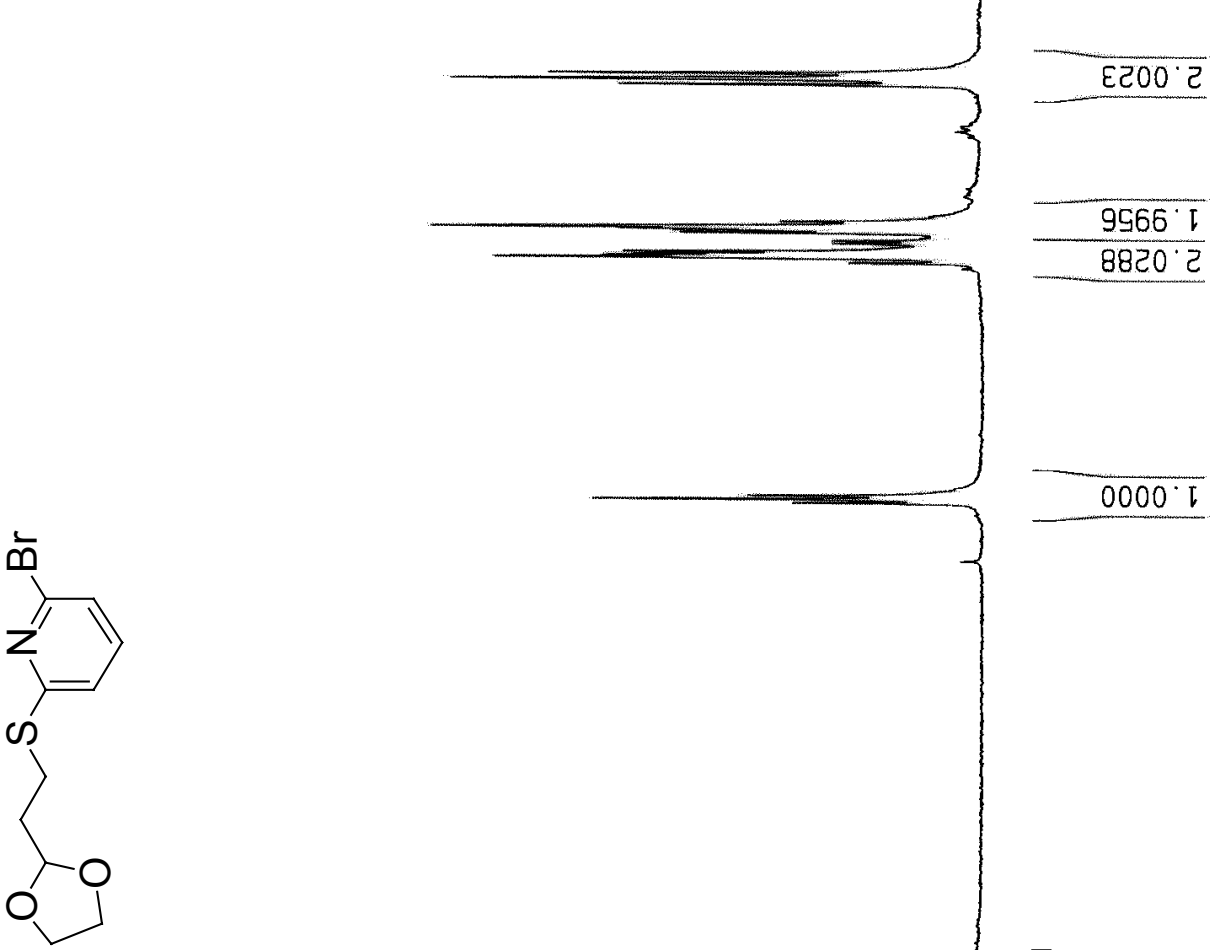

-m

I $900 E^{\prime} L$

I $\triangle$ GEE ' $\angle$

EटटGE ' $L$

wdd
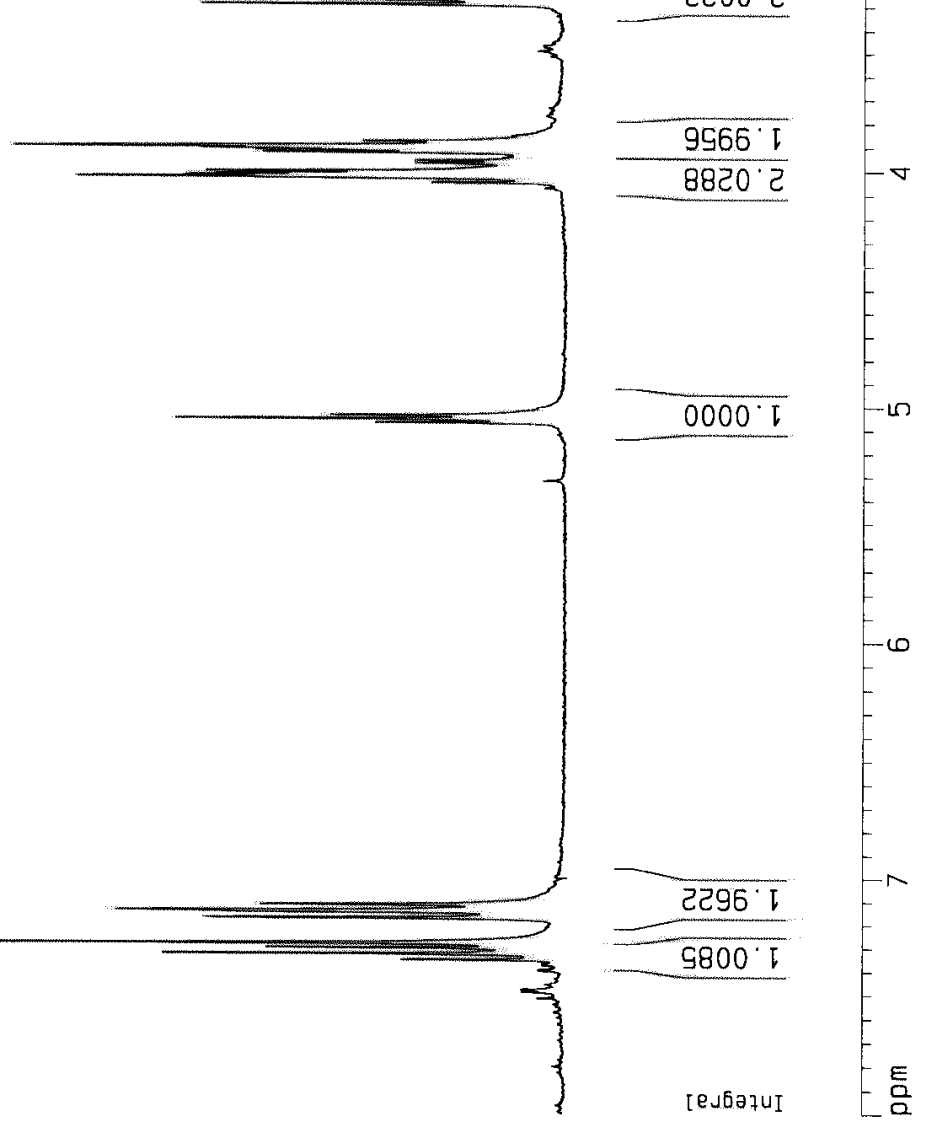

\section{S-49}


<smiles>CCOC(=O)OCC</smiles>

$6 \angle E^{\prime} 99$

$200^{\circ} \angle L$
$92 \nabla^{\circ} \angle L$

$6 \triangleright 8 \cdot \angle L$

$\angle S 9^{\circ} E O T$

I66.02 -

$609^{\circ} \varepsilon 己 \tau$

I6C $8 E$ !

I86 IDT

$\nabla L L^{\circ} 097$

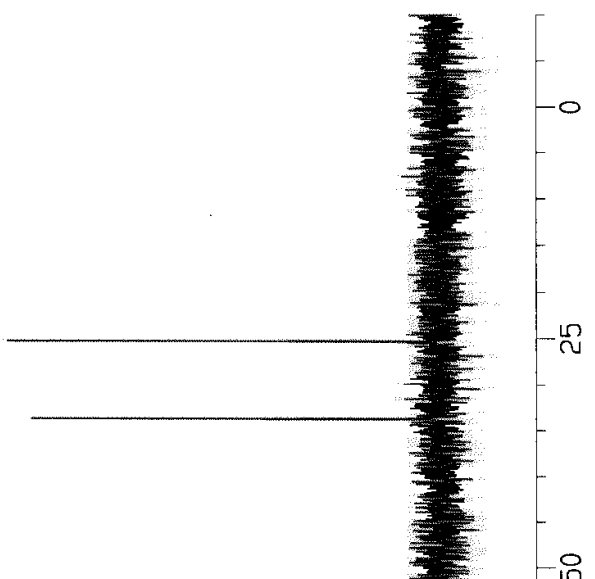




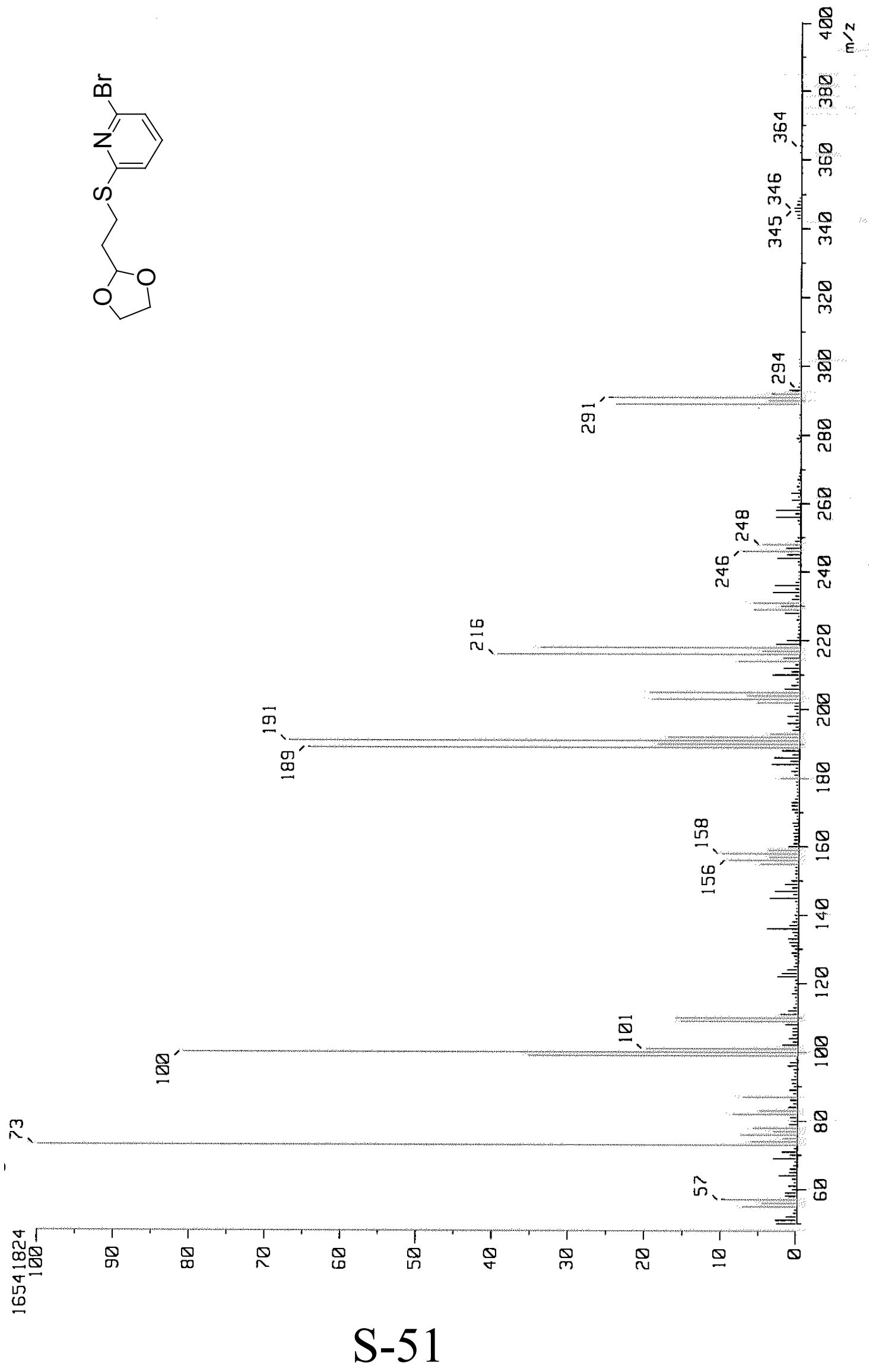

